\title{
Differential gene expression profiling of endometrium during the mid-luteal phase of the estrous cycle between a repeat breeder (RB) and non-RB cows
}

Ken-Go Hayashi ${ }^{1}$, Misa Hosoe ${ }^{2}$, Keiichiro Kizaki ${ }^{3}$, Shiori Fujii ${ }^{1}$, Hiroko Kanahara ${ }^{1}$, Toru Takahashi ${ }^{3}$ and Ryosuke Sakumoto ${ }^{1 *}$

\begin{abstract}
Background: Repeat breeding directly affects reproductive efficiency in cattle due to an increase in services per conception and calving interval. This study aimed to investigate whether changes in endometrial gene expression profile are involved in repeat breeding in cows. Differential gene expression profiles of the endometrium were investigated during the mid-luteal phase of the estrous cycle between repeat breeder (RB) and non-RB cows using microarray analysis.
\end{abstract}

Methods: The caruncular (CAR) and intercaruncular (ICAR) endometrium of both ipsilateral and contralateral uterine horns to the corpus luteum were collected from RB (inseminated at least three times but not pregnant) and non-RB cows on Day 15 of the estrous cycle (4 cows/group). Global gene expression profiles of these endometrial samples were analyzed with a $15 \mathrm{~K}$ custom-made oligo-microarray for cattle. Immunohistochemistry was performed to investigate the cellular localization of proteins of three identified transcripts in the endometrium.

Results: Microarray analysis revealed that 405 and 397 genes were differentially expressed in the CAR and ICAR of the ipsilateral uterine horn of RB, respectively when compared with non-RB cows. In the contralateral uterine horn, 443 and 257 differentially expressed genes were identified in the CAR and ICAR of RB, respectively when compared with non-RB cows. Gene ontology analysis revealed that genes involved in development and morphogenesis were mainly up-regulated in the CAR of RB cows. In the ICAR of both the ipsilateral and contralateral uterine horns, genes related to the metabolic process were predominantly enriched in the RB cows when compared with non-RB cows. In the analysis of the whole uterus (combining the data above four endometrial compartments), RB cows showed up-regulation of 37 genes including PRSS2, GSTA3 and PIPOX and down-regulation of 39 genes including CHGA, KRT35 and THBS4 when compared with non-RB cows. Immunohistochemistry revealed that CHGA, GSTA3 and PRSS2 proteins were localized in luminal and glandular epithelial cells and stroma of the endometrium.

Conclusion: The present study showed that endometrial gene expression profiles are different between RB and non-RB cows. The identified candidate endometrial genes and functions in each endometrial compartment may contribute to bovine reproductive performance.

Keywords: Repeat breeder, Endometrium, Caruncle, Intercaruncle, Microarray, Cow

\footnotetext{
* Correspondence: sakumoto@affrc.go.jp

${ }^{1}$ Division of Animal Breeding and Reproduction Research, Institute of

Livestock and Grassland Science, National Agriculture and Food Research

Organization, Tsukuba 305-0901, Japan

Full list of author information is available at the end of the article
} 


\section{Background}

Repeat breeder (RB) is generally defined as any cow that has failed to conceive after at least three inseminations. In both dairy and beef cattle herds, the presence of $\mathrm{RB}$ cows can directly lead a large economic loss for producers due to an extension of the length of the open period and frequent artificial insemination (AI) [1]. In addition to management problems such as inadequate estrus detection and AI techniques, various physiological problems of individual cows are one of major causes of repeat breeding. For example, infections of uterus, cervix and/or vagina, dysfunctions of uterus or ovary, obstructed oviducts, defective oocytes and anatomical defects of the reproductive tracts are involved in conception failure, early embryonic death and endocrine disorders of $\mathrm{RB}$ animals. [1]. It has been reported that embryo transfer is effective to improve the fertility of $R B$ cows and heifers $[2,3]$. On the other hand, a study of reciprocal transfers of embryos between RB and virgin heifers showed that a higher proportion of embryos transferred from $\mathrm{RB}$ to virgin heifers than from virgin to $\mathrm{RB}$ heifers survived at day 16 to 17 , suggesting that the uterine environment in RB heifers is less suitable than in the virgins for supporting a successful embryo development [4]. This became more evident by transfer of identical demi-embryos to $\mathrm{RB}$ and virgin recipient heifers resulted less number of morphologically normal and elongated embryos in the RB heifers than in the virgin heifers at day 15 [5]. About an association between alteration of uterine environment and repeat breeding, Katagiri et al. have demonstrated that there is a close relationship between the endometrial epidermal growth factor profile and diminished fertility of RB cows [6].

The molecular mechanisms underlying endometrial function may contribute to reproductive performance in cattle. Increasing evidence using global gene expression analysis has identified numerous differentially expressed genes and related functional pathways in bovine endometrium among highly fertile, subfertile and infertile animal strains during estrous cycle or early pregnancy [7-10]. Recent studies have also investigated gene expression profiles under various conditions of the bovine endometrium during the estrous cycle and/or during early pregnancy using DNA microarray or RNA sequencing [11-18]. In addition, microarray studies have revealed that heat stress and steroid hormones directly affect bovine endometrial gene expression profiles [19, 20].

In ruminants, the endometrium shows structural and physiological differences depending on the uterine compartments. The caruncular (CAR) areas are aglandular and a limited area that forms placentomes by fusing with the fetal extraembryonic membrane [21, 22]. On the other hand, the intercaruncular (ICAR) areas contain endometrial glands that synthesize and secrete substances or factors that are essential for survival and development of the conceptus [23, 24]. A study that directly compared the gene expression profiles of CAR and ICAR during implantation in cows showed 1177 and 453 differentially expressed genes (DEG) were found for cyclic and pregnant animals, respectively [13]. In addition, it has been reported that tissues of the ipsilateral uterine horn to the ovary with the corpus luteum (CL) contain greater quantities of progesterone (P4) and are more sensitive to $\mathrm{P} 4$ as compared with tissues on the contralateral side [25]. Although a previous study demonstrated that a few genes show differences in expression between ipsilateral and contralateral uterine horns during the bovine estrous cycle [11], we consider that it is important to analyze each compartment of the bovine endometrium separately in order to understand enodometrial function more comprehensively.

These previous studies suggest that alteration of the endometrial function due to changes in gene expression may contribute to their lower reproductive performance in RB cows, whereas details of the molecular mechanisms and biological pathways of their endometria still need to be elucidated. Thus, we hypothesized that there is a characteristic gene expression profile in the endometrium of the RB cows. This study aimed to investigate differences in gene expression profiles of the endometrium between $\mathrm{RB}$ and non- $\mathrm{RB}$ cows during the midluteal phase of the estrous cycle. In pregnant cattle, maternal recognition of pregnancy occurs around Day 14-15 [26]. In addition, it has been reported that the majority of early embryo losses in cattle have occurred within 16 days of gestation (i.e. during the mid-luteal phase) $[27,28]$. Therefore, the basal gene expression profiles of endometrium at mid luteal phase would have the most important association with reproductive performance.

\section{Methods}

\section{Animals and sample collection}

This study was carried out using non-lactating Japanese Black cows at the institute's ranch (age: $7.8 \pm 0.9$ years, parity: $3.3 \pm 0.8$, open period from last parturition to first AI in this study: $104 \pm 9.6$ month). Repeat breeder cows $(n=4)$ were defined based on a previous study by Dochi et al. [3]. Briefly, the RB cows had three characteristics as follows: (1) detectable estrous behavior, but not always normal estrous cycles; (2) not conceiving after three or more inseminations following normal estrous behavior; and (3) healthy uterus and ovaries, as determined by transrectal palpation. Non-RB cows $(n=4)$ conceived within three inseminations. The non-RB cows were confirmed to be pregnant by transrectal ultrasonography (HS-1500V; Honda Electronics. Co., Aichi, 
Japan) at 40 days after insemination, then abortion was induced by a single intramuscular injection of $500 \mu \mathrm{g}$ of prostaglandin F2 $\alpha$ (cloprostenol [Dalmazin]; Kyoritsu Seiyaku. Co., Tokyo, Japan) followed by repeated normal estrous cycles at least twice. Both RB and non-RB cows were slaughtered on Day 15 of the estrous cycle (the day of estrus was designated as Day 0) and the uterus and both ovaries together were collected. Uterine horns were identified as ipsilateral to the ovary containing the CL or contralateral. We collected CAR and ICAR in the endometrium from the middle area of each uterine horns. The uterine horns were cut opened longitudinally using scissors and CAR were carefully dissected first not to include ICAR, subsequently, ICAR areas were cut off. Collected samples were snap-frozen in liquid nitrogen and stored at $-80{ }^{\circ} \mathrm{C}$ until RNA extraction. Whole cross section of the uterus for immunohistochemistry were collected from the middle area of ipsilateral uterine horn of all cows and fixed in $10 \%$ formalin $(\mathrm{v} / \mathrm{v})$, embedded in paraffin wax, and then stored at $4{ }^{\circ} \mathrm{C}$ until use. All procedures in animal experiments were carried out in accordance with guidelines approved by the Animal Ethics Committee of the National Institute of Agrobiological Sciences for the use of animals (permission number: H18-036).

\section{Microarray analysis}

Total RNA was extracted from each sample by acid guanidinium thiocyanate-phenol-chloroform with ISOGEN (Nippon Gene, Tokyo, Japan) according to the manufacturer's instructions. All RNA samples were then treated with TURBO DNase (TURBO DNA-free ${ }^{\text {тм }}$ Kit, Thermo Fisher Scientific, Waltham, MA, USA) according to the manufacturer's instructions to remove contaminating genomic DNA. The quantity and quality of the total RNA samples were assessed using a NanoDrop spectrophotometer (ND-1000; NanoDrop Technology Inc., Wilmington, DE, USA) and an Experion automated electrophoresis system with an Experion RNA StdSens kit (Bio-Rad Laboratories, Hercules, CA, USA), respectively. A custom-made bovine oligonucleotide microarray with 15,000 unique genes (GPL9284) fabricated by Agilent Technologies (Santa Clara, CA, USA) was used in this study, which was performed as described previously [29]. Sixty-mer nucleotide probes for the customized microarray were synthesized on a glass slide. We performed one-color microarray analysis. cDNA synthesis, Cy3-labeled cRNA preparation, hybridization, and the washing and scanning of array slides were performed according to the Agilent one color microarray-based gene expression analysis protocol. Briefly, $400 \mathrm{ng}$ of total RNA from each sample were reverse-transcribed into cDNA using the Quick Amp Labeling Kit (Agilent Technologies) with an oligo dT-based primer, and then
Cy3-labelled cRNA was prepared by in vitro transcription. Labeled cRNA was purified with an RNeasy Mini Kit (Qiagen, Hilden, Germany), and the concentration and $\mathrm{Cy} 3$ dye incorporation (pmol $\mathrm{Cy} 3 / \mu \mathrm{g}$ cRNA) were measured with a spectrophotometer. Labeled cRNA (600 ng) was fragmented and hybridized using the Gene Expression Hybridization Kit (Agilent Technologies), according to the manufacturer's instructions. The arrays were washed using a Gene Expression Wash Pack Kit (Agilent Technologies) and scanned using an Agilent Microarray Scanner. Feature Extraction ver. 9.5 was used for image analysis and data extraction. Microarray data from each sample were imported into GeneSpring 12 (Agilent Technologies) for further data characterization. The GEO accession numbers are as follows. Platform: GPL9284; samples: GSM2093338 to GSM2093369; series: GSE79367. To identify putative biological functions of DEG between RB and non-RB cows in each endometrial compartment, we performed functional annotation chart analysis of the lists of DEG using the Database for Annotation, Visualization and Integrated Discovery (DAVID; http://david.abcc.ncifcrf.gov/) based on Genebank Accession IDs [30]. Gene Ontology (GO) Biological Process was selected as the functional annotation category for the analysis with the threshold for minimum gene counts belonging to an annotation term set to 5 and an EASE score set to 0.05. The GO terms were ranked according to their $P$-values describing the significance of gene-term enrichment.

\section{Quantitative real-time RT-PCR analysis}

To validate the results of microarray analysis, we confirmed mRNA expression of the following representative genes using quantitative real-time RT-PCR (qPCR) analysis: (1) top two up- or down-regulated known genes in each endometrial compartment; and (2) top five upor down-regulated known genes in the whole uterus. Details of the procedures for single-strand cDNA synthesis and qPCR were previously described [31]. Briefly, 50 ng of total RNA from the same sample used for the microarray were reverse-transcribed into cDNA for $30 \mathrm{~min}$ at $48{ }^{\circ} \mathrm{C}$ using MultiScribe ${ }^{\mathrm{TM}}$ Reverse Transcriptase (Applied Biosystems, Foster City, CA, USA) with a random primer, dNTP mixture, $\mathrm{MgCl}_{2}$ and RNase inhibitor. After heat inactivation of the reverse transcriptase for $5 \mathrm{~min}$ at $95{ }^{\circ} \mathrm{C}, \mathrm{PCR}$ and resulting relative increase in reporter fluorescent dye emission were monitored in real time using an Mx3000P qPCR system (Agilent Technologies). Primers were designed using Primer Express computer software program (Applied Biosystems) or Primer3 Plus software (www.bioinformatics.nl/primer3plus/) based on the bovine sequences. The primer sequences for each gene are listed in Table 1. Thermal-cycling conditions included an initial sample incubation at $50{ }^{\circ} \mathrm{C}$ for $2 \mathrm{~min}$ and 
Table 1 Details of the primers used for quantitative real-time RT-PCR analysis

\begin{tabular}{|c|c|c|c|}
\hline Gene (GenBank accession number) & Primer & Sequence & Position \\
\hline$\overline{C H G A}$ & Forward & 5'-GCCGAAAGAGGTGACAGAAGA-3' & $538-558$ \\
\hline (NM_181005) & Reverse & 5'-GTCTCCGTCCGAGTCTTCATC-3' & $637-617$ \\
\hline CNGA1 & Forward & 5'-AGCAGAGATCGCCATCAATGT-3' & 1574-1594 \\
\hline (NM_174278) & Reverse & 5'-ACCAACTCCACCAACAGACCA-3' & $1663-1643$ \\
\hline CPXM2 & Forward & 5'- ACCAGTGGATTGAAGTGGACG-3' & $581-601$ \\
\hline (NM_001206057) & Reverse & 5'- TCACTCAGCCAGAGTGAGTTCCT-3' & $665-643$ \\
\hline FAM83D & Forward & 5'- GGCTCCTACAGTITTACATGGACAG-3' & $788-812$ \\
\hline (NM_001083393) & Reverse & 5'-CAACCACTTGGCCAGACAGAA-3' & $863-843$ \\
\hline FMO2 & Forward & 5'- AAGCCAGACATCCTITCTCTCTTG -3' & $1459-1482$ \\
\hline (NM_001163274) & Reverse & 5'- CCCAACCAGGCGATACTGATA-3' & 1554-1532 \\
\hline GSTA3 & Forward & 5'-AGAGCCATCCTCAGCTACCTTG-3' & $254-275$ \\
\hline (NM_001077112) & Reverse & 5'-TCGATCCTGACTGTCTCCTTCA-3' & $327-306$ \\
\hline$|F| H 1$ & Forward & 5'-GGGACTAACAGCTTCACCAGGT-3' & 1764-1785 \\
\hline (XM_002685338) & Reverse & 5'-GGTAACTGCATCAAGATTGGCA-3' & 1860-1839 \\
\hline IGGIC & Forward & 5'-ACCAAGGTGGACAAGGCTGTT-3' & $274-294$ \\
\hline (S82409) & Reverse & 5'-GGAAGATGAAGACAGAGGGTCCT-3' & $370-348$ \\
\hline KCNA2 & Forward & 5'-TGGGTTCCCTATGTGCAATTG-3' & 1644-1664 \\
\hline (NM_001101195) & Reverse & 5'-TCCCGGTGGTAGAAGTAGTTGAA-3' & $1734-1712$ \\
\hline KLHL24 & Forward & 5'- TTATTGGCAAGGAGGAGATGGT-3' & $901-922$ \\
\hline (NM_001206196) & Reverse & 5'- TCTCAGATCAACAGCGCGAT-3' & $968-949$ \\
\hline KRT35 & Forward & 5'- GAGACCGAGGTATCCATGCG-3' & $587-606$ \\
\hline (NM_001076073) & Reverse & 5'- TTCTTGAGGCAGAGCAGCTC -3' & $726-707$ \\
\hline LPLUNC1 & Forward & 5'- TCGGTGTGTTCAACCCTAAGC-3' & $1280-1300$ \\
\hline (NM_174697) & Reverse & 5'- TTCTCGTTTGGCAGCAGGAT -3' & $1355-1336$ \\
\hline PIPOX & Forward & 5'- ACAGCATTAACACCGAGTCGG-3' & $2140-2160$ \\
\hline (NM_001014878) & Reverse & 5'- GGCAGTTATGAGCCTGTTTCCT-3' & $2210-2189$ \\
\hline PLEKHA5 & Forward & 5'- GATGGATTCAAGAACGGAACG-3' & $2655-2675$ \\
\hline (XM_002687754) & Reverse & 5'- TTCCACAGTCATCCTAGGTCGA-3' & $2739-2718$ \\
\hline PRF1 & Forward & 5'-CAAGCCAAATGCTAATGTCCGT-3' & $408-429$ \\
\hline (NM_001143735) & Reverse & 5'-AAAGCGACACTCCACTAAGTCCAT-3' & $531-508$ \\
\hline PRSS2 & Forward & 5'-GTGAGGCTGGGAGAATACAACA-3' & $211-232$ \\
\hline (NM_174690) & Reverse & 5'-ATGATCTTGGACGCATCGATGA-3' & $281-260$ \\
\hline SLC39A2 & Forward & 5'- TTGGCTGCCTATTTGCCCT-3' & $355-373$ \\
\hline (NM_001205648) & Reverse & 5'- CTGGAACCACTTGAAGCAGATG-3' & $428-407$ \\
\hline THBS4 & Forward & 5'- CACTCTGAACGAGCTCTACGTGAT 3' & $331-354$ \\
\hline (NM_001034728) & Reverse & 5'- GAAGAGTAAAGGCCGAAGATGGT-3' & 411-389 \\
\hline SUZ12 & Forward & 5'-GAACACCTATCACACACATTCTTGT-3' & $1565-1589$ \\
\hline (NM_001205587) & Reverse & 5'-TAGAGGCGGTTGTGTCCACT-3' & $1694-1675$ \\
\hline
\end{tabular}

at $95{ }^{\circ} \mathrm{C}$ for $10 \mathrm{~min}$, followed by 40 cycles at $95{ }^{\circ} \mathrm{C}$ for $15 \mathrm{~s}$ and at $60{ }^{\circ} \mathrm{C}$ for $1 \mathrm{~min}$. The cycle threshold value $\left(\mathrm{C}_{\mathrm{T}}\right)$ indicate the quantity of the target gene in each sample. The relative difference in initial amount of each mRNA species (or cDNA) was determined by comparing the $C_{T}$ values. The standard curves for each gene were generated by serially diluting plasmids containing cDNA of each individual gene to quantify the mRNA concentrations. We confirmed the utility of the dissociation curve for detecting the SYBR Green-based objective amplicon because SYBR Green also detects double-stranded DNA including Primer dimers, contaminating DNA and PCR products 
Table 2 Top 10 up- and down-regulated known genes in CAR of ipsilateral uterine horns of RB cows

\begin{tabular}{|c|c|c|c|c|}
\hline GenBank accession ID & Gene symbol & Gene description & Fold change & $P$-value \\
\hline \multicolumn{5}{|l|}{ Up-regulated genes } \\
\hline NM_001077112 & GSTA3 & Glutathione S-transferase, alpha 3 & 19.2 & 0.0016 \\
\hline NM_001206196 & KLHL24 & Kelch-like 24 (Drosophila) & 3.0 & 0.0273 \\
\hline XM_588022 & SPOPL & Speckle-type POZ protein-like & 2.8 & 0.0239 \\
\hline NM_001103317 & ERCC2 & Excision repair cross-complementing rodent repair deficiency, complementation group 2 & 2.5 & 0.0437 \\
\hline XM_002696037 & CD300LG & CD300 molecule-like family member $\mathrm{g}$ & 2.2 & 0.0378 \\
\hline NM_001075908 & STK33 & Serine/threonine kinase 33 & 2.1 & 0.0351 \\
\hline NM_174607 & SLC5A3 & Solute carrier family 5 (inositol transporters), member 3 & 2.0 & 0.0126 \\
\hline NM_001192523 & KCNMB4 & Potassium large conductance calcium-activated channel, subfamily M, beta member 4 & 2.0 & 0.0307 \\
\hline NM_001083638 & MEF2A & Myocyte enhancer factor $2 \mathrm{~A}$ & 2.0 & 0.0290 \\
\hline XM_002695445 & ZNF211 & Zinc finger protein 211 & 2.0 & 0.0063 \\
\hline \multicolumn{5}{|l|}{ Down-regulated genes } \\
\hline NM_001206057 & CPXM2 & Carboxypeptidase X (M14 family), member 2 & 5.3 & 0.0496 \\
\hline NM_001076073 & KRT35 & Keratin 35 & 4.1 & 0.0279 \\
\hline NM_001101239 & GRP & Gastrin-releasing peptide & 3.6 & 0.0319 \\
\hline NM_001245926 & FGF9 & Fibroblast growth factor 9 & 3.5 & 0.0066 \\
\hline NM_174145 & PKP1 & Plakophilin 1 (ectodermal dysplasia/skin fragility syndrome) & 2.9 & 0.0021 \\
\hline NM_001076864 & TMEM129 & Transmembrane protein 129 & 2.6 & 0.0087 \\
\hline NM_001105478 & SSLP1 & Secreted seminal-vesicle Ly-6 protein 1 & 2.5 & 0.0474 \\
\hline NM_001077962 & STAC & $\mathrm{SH} 3$ and cysteine rich domain & 2.4 & 0.0157 \\
\hline NM_001077945 & PFN3 & Profilin 3 & 2.4 & 0.0106 \\
\hline NM_001012685 & FCAR & Fc fragment of IgA, receptor for & 2.3 & 0.0322 \\
\hline
\end{tabular}

from misannealed primers. Non-specific amplicons appear as a peak separate from the desired amplicon peak. The expression ratio of each gene to SUZ12 mRNA, which has been demonstrated to be suitable for normalization in bovine endometrial tissue [32], was calculated to adjust for any variations in the $\mathrm{qPCR}$ reaction.

\section{Immunohistochemistry}

Immunohistochemistry for chromogranin A (CHGA), glutathione $S$-transferase A3 (GSTA3) and trypsin 2 (PRSS2) was performed in the endometrium of both RB and non-RB cows on Day 15 of the estrous cycle using the automated Ventana HX System Discovery with a DabMapKit (Roche Diagnostics, Basel, Switzerland) as described previously in detail by our laboratory [33]. Uterine cross sections $7-\mu \mathrm{m}$-thick were incubated at room temperature with rabbit polyclonal anti-human CHGA antibody $(1.0 \mathrm{mg} / \mathrm{ml}, 20085$, ImmunoStar Inc., Hudson, WI, USA), rabbit polyclonal anti-human GSTA3 antibody (0.5 mg/ml, orb5362, Biorbyt LLC, San Francisco, CA, USA) or rabbit polyclonal anti-bovine PRSS2 antibody (10 mg/ml, OASA07087, Aviva Systems Biology, San Diego, CA, USA) diluted 1:100 (anti-CHGA), 1:20 (antiGSTA3) or 1:200 (anti-PRSS2) in Discovery Ab diluents (Roche) for $12 \mathrm{~h}$. The signals were detected using anti- rabbit IgG-Biotin conjugate (Sigma) diluted 1:500 for $1 \mathrm{~h}$. Negative controls were performed using normal rabbit IgG (0.5 mg/ml, 20304, Imgenex, San Diego, CA, USA) diluted at concentrations equivalent to the primary antibodies. The sections were observed with a Leica DMRE HC microscope (Leica Microsystems, Wetzlar, Germany)

Table 3 Top 5 functional annotations of up- and down-regulated genes in CAR of ipsilateral uterine horns

\begin{tabular}{lll}
\hline Term & Count & $P$-value \\
\hline Up-regulated genes & & \\
GO:0048856 anatomical structure development & 11 & 0.0029 \\
GO:0032502 developmental process & 11 & 0.0161 \\
GO:0009987 cellular process & 31 & 0.0186 \\
GO:0007275 $\sim$ multicellular organismal development & 10 & 0.0230 \\
GO:0009888 tissue development & 5 & 0.0246 \\
Down-regulated genes & & \\
GO:0009987 cellular process & 95 & $<0.0001$ \\
GO:0007010 cytoskeleton organization & 8 & 0.0061 \\
GO:0022610 biological adhesion & 11 & 0.0065 \\
GO:0007155 cell adhesion & 11 & 0.0065 \\
GO:0016043 cellular component organization & 23 & 0.0099 \\
\hline
\end{tabular}


Table 4 Top 10 up- and down-regulated known genes in ICAR of ipsilateral uterine horns of RB cows

\begin{tabular}{|c|c|c|c|c|}
\hline GenBank accession ID & Gene symbol & Gene description & Fold change & $P$-value \\
\hline \multicolumn{5}{|l|}{ Up-regulated genes } \\
\hline NM_174697 & LPLUNC1 & Von Ebner minor salivary gland protein & 3.7 & 0.0214 \\
\hline NM_001075162 & FMO2 & Flavin containing monooxygenase 2 (non-functional) & 3.3 & 0.0348 \\
\hline NM_001166616 & C5 & Complement component 5 & 3.2 & 0.0429 \\
\hline XM_002692160 & FOXA2 & Forkhead box A2 & 3.0 & 0.0350 \\
\hline NM_181027 & AKR1C4 & $\begin{array}{l}\text { Aldo-keto reductase family 1, member C4 (chlordecone reductase; 3-alpha } \\
\text { hydroxysteroid dehydrogenase, type l; dihydrodiol dehydrogenase 4) }\end{array}$ & 2.9 & 0.0104 \\
\hline NM_001045878 & GATM & Glycine amidinotransferase (L-arginine:glycine amidinotransferase) & 2.8 & 0.0472 \\
\hline NM_001206196 & KLHL24 & Kelch-like 24 (Drosophila) & 2.6 & 0.0301 \\
\hline NM_001034419 & HPGD & Hydroxyprostaglandin dehydrogenase 15-(NAD) & 2.6 & 0.0293 \\
\hline XM_001254052 & ZNED1 & DNA-directed RNA polymerase I subunit RPA12-like & 2.4 & 0.0476 \\
\hline NM_001038096 & $\mathrm{CFI}$ & Complement factor I & 2.4 & 0.0096 \\
\hline \multicolumn{5}{|l|}{ Down-regulated genes } \\
\hline NM_001034728 & THBS4 & Thrombospondin 4 & 3.4 & 0.0106 \\
\hline NM_001083393 & FAM83D & Protein FAM83D & 2.6 & 0.0011 \\
\hline NM_001105411 & GFRA1 & GDNF family receptor alpha 1 & 2.4 & 0.0391 \\
\hline NM_001206057 & CPXM2 & Carboxypeptidase X (M14 family), member 2 & 2.3 & 0.0231 \\
\hline NM_178572 & CA2 & Carbonic anhydrase II & 2.3 & 0.0474 \\
\hline NM_001099381 & GALK1 & Galactokinase 1 & 2.1 & 0.0466 \\
\hline NM_001035050 & VTN & Vitronectin & 2.0 & 0.0464 \\
\hline NM_174745 & MMP2 & Matrix metallopeptidase 2 (gelatinase A, 72 kDa gelatinase, 72 kDa type IV collagenase) & 1.9 & 0.0387 \\
\hline NM_001075730 & STRA6 & Stimulated by retinoic acid gene 6 & 1.9 & 0.0405 \\
\hline NM_174558 & KCNK17 & Potassium channel, subfamily K, member 17 & 1.9 & 0.0496 \\
\hline
\end{tabular}

and a Nikon Digital Sight DS-Fi1-L2 (Nikon Instruments Co., Tokyo, Japan).

\section{Statistical analysis}

Microarray data were analyzed statistically with an unpaired Student's $t$-test and summarized using GeneSpring 12 (Agilent Technologies). The analysis of each uterine compartment was performed by comparing the gene datasets which composed by microarray data of four cows in each $\mathrm{RB}$ and non-RB group ( $n=4$ /group). The analysis of whole uterus was performed by comparing the gene datasets which composed by microarray data of all four compartments of four cows in each RB and non-RB group ( $n=16$ /group). The qPCR results were analyzed using a Mann-Whitney $U$ test. Results are presented as the mean \pm SEM. Statistical significance is considered to be at $P<0.05$.

\section{Results}

Gene expression profiles of CAR and ICAR in ipsilateral uterine horns

A total of 405 and 397 genes were differentially expressed in CAR and ICAR of the ipsilateral uterine horn of $R B$ cows, respectively when compared with non-RB cows (adjusted $P$-value $<0.05$, fold-change $>1.0$ ). All data of individual gene changes in CAR and ICAR are available in Additional file 1: Tables S1 and S2, respectively. Out of these, 128 genes were up-regulated and 277 genes were down-regulated in CAR, whereas 169 genes were upregulated and 228 genes were down-regulated in ICAR. The top 10 up- and down-regulated known genes in CAR

Table 5 Top 5 functional annotations of up- and down-regulated genes in ICAR of ipsilateral uterine horns

\begin{tabular}{lll}
\hline Term & Count & $P$-value \\
\hline Up-regulated genes & & \\
GO:0008152 metabolic process & 38 & 0.0033 \\
GO:0044237 cellular metabolic process & 29 & 0.0242 \\
GO:0044249 cellular biosynthetic process & 15 & 0.0345 \\
GO:0048878 chemical homeostasis & 5 & 0.0423 \\
Down-regulated genes & & \\
G0:0008152 metabolic process & 66 & $<0.0001$ \\
GO:0044237 cellular metabolic process & 8 & $<0.0001$ \\
G0:0009987 cellular process & 11 & 0.0001 \\
GO:0044238 primary metabolic process & 11 & 0.0009 \\
G0:0019538 protein metabolic process & 23 & 0.0023 \\
\hline
\end{tabular}


Table 6 Top 10 up- and down-regulated known genes in CAR of contralateral uterine horns of RB cows

\begin{tabular}{|c|c|c|c|c|}
\hline GenBank accession ID & Gene symbol & Gene description & Fold change & $P$-value \\
\hline \multicolumn{5}{|l|}{ Up-regulated genes } \\
\hline NM_001077112 & GSTA3 & Glutathione S-transferase, alpha 3 & 12.7 & 0.0080 \\
\hline NM_001014878 & PIPOX & Pipecolic acid oxidase & 8.4 & 0.0261 \\
\hline NM_001024569 & ELF5 & E74-like factor 5 (ets domain transcription factor) & 4.3 & 0.0173 \\
\hline NM_173981 & ACAN & Aggrecan & 3.0 & 0.0420 \\
\hline NM_174404 & NRXN1 & Neurexin 1 & 3.0 & 0.0065 \\
\hline NM_001079771 & SMOC1 & SPARC related modular calcium binding 1 & 2.7 & 0.0104 \\
\hline NM_001034351 & TNNC1 & Troponin C type 1 (slow) & 2.6 & 0.0142 \\
\hline NM_173945 & NTS & Neurotensin & 2.6 & 0.0289 \\
\hline NM_001206196 & KLHL24 & Kelch-like 24 (Drosophila) & 2.4 & 0.0345 \\
\hline NM_001046585 & CCL14 & Chemokine (C-C motif) ligand 14 & 2.4 & 0.0358 \\
\hline \multicolumn{5}{|l|}{ Down-regulated genes } \\
\hline NM_001205648 & SLC39A2 & Solute carrier family 39 (zinc transporter), member 2 & 2.7 & 0.0110 \\
\hline XM_002687754 & PLEKHA5 & Pleckstrin homology domain containing, family A member 5 & 2.2 & 0.0181 \\
\hline NM_001077962 & STAC & $\mathrm{SH} 3$ and cysteine rich domain & 2.0 & 0.0456 \\
\hline NM_001098061 & SQLE & Squalene epoxidase & 2.0 & 0.0268 \\
\hline NM_174145 & PKP1 & Plakophilin 1 (ectodermal dysplasia/skin fragility syndrome) & 1.9 & 0.0304 \\
\hline NM_001098938 & CYP39A1 & Cytochrome P450, family 39, subfamily A, polypeptide 1 & 1.9 & 0.0262 \\
\hline NM_174489 & VLDLR & Very low density lipoprotein receptor & 1.9 & 0.0063 \\
\hline NM_001034660 & SLC5A11 & Solute carrier family 5 (sodium/glucose cotransporter), member 11 & 1.8 & 0.0061 \\
\hline NM_001075803 & $\mathrm{FH}$ & Fumarate hydratase & 1.8 & 0.0009 \\
\hline NM_001099399 & CMTM3 & CKLF-like MARVEL transmembrane domain containing 3 & 1.8 & 0.0434 \\
\hline
\end{tabular}

are shown in Table 2. The most pronounced up- and down-regulation of gene expression in $\mathrm{RB}$ cows was observed for GSTA3 (Glutathione S-transferase, alpha 3; 19.2-fold) and CPXM2 (Carboxypeptidase X (M14 family), member 2; 5.3-fold), respectively. The top five functional annotations of DEG in the CAR of ipsilateral uterine horns between RB and non-RB cows are listed in Table 3. The GO terms involved in anatomical structure development, developmental process, cellular process, multicellular organismal development and biosynthetic process were highly enriched in up-regulated genes, whereas the GO terms involved in cellular process, cytoskeleton organization, biological adhesion, cell adhesion and cellular component organization were highly enriched in down-regulated genes.

The top 10 up- and down-regulated known genes in ICAR are shown in Table 4. The highest increase and decrease in gene expression in RB cows were observed in LPLUNC1 (Von Ebner minor salivary gland protein; 3.7-fold) and THBS4 (Thrombospondin 4; 3.4fold), respectively. Table 5 summarizes the top five functional annotations of DEG in ICAR between RB and non-RB cows. As a result of DAVID analysis, only four GO terms related to metabolic process, cellular metabolic process, cellular biosynthetic process and chemical homeostasis were identified in upregulated genes. In down-regulated genes, the GO terms involved in metabolic process, cellular metabolic process, cellular process, primary metabolic process and protein metabolic process were highly enriched.

Table 7 Top 5 functional annotations of up- and down-regulated genes in CAR of contralateral uterine horns

\begin{tabular}{lll}
\hline Term & Count & $P$-value \\
\hline Up-regulated genes & & \\
GO:0048518 positive regulation of biological process & 25 & $<0.0001$ \\
GO:0048522 positive regulation of cellular process & 22 & $<0.0001$ \\
GO:0009887 organ morphogenesis & 12 & $<0.0001$ \\
GO:0009653 anatomical structure morphogenesis & 16 & 0.0001 \\
GO:0048856 anatomical structure development & 24 & 0.0002 \\
Down-regulated genes & & \\
GO:0045859 regulation of protein kinase activity & 5 & 0.0029 \\
GO:0043549 regulation of kinase activity & 5 & 0.0035 \\
GO:0051338 regulation of transferase activity & 5 & 0.0040 \\
GO:0043436 oxoacid metabolic process & 7 & 0.0075 \\
GO:0019752 carboxylic acid metabolic process & 7 & 0.0075 \\
\hline
\end{tabular}


Table 8 Top 10 up- and down-regulated known genes in ICAR of contralateral uterine horns of RB cows

\begin{tabular}{|c|c|c|c|c|}
\hline GenBank accession ID & Gene symbol & Gene description & Fold change & $P$-value \\
\hline \multicolumn{5}{|l|}{ Up-regulated genes } \\
\hline NM_001014878 & PIPOX & Pipecolic acid oxidase & 8.8 & 0.0156 \\
\hline NM_174278 & CNGA1 & Cyclic nucleotide gated channel alpha 1 & 6.8 & 0.0390 \\
\hline NM_001033608 & GSTA3 & Glutathione S-transferase, alpha 3 & 6.6 & 0.0340 \\
\hline NM_001046400 & MIF & Macrophage migration inhibitory factor (glycosylation-inhibiting factor) & 3.1 & 0.0118 \\
\hline NM_001046400 & ZNRD1 & Zinc ribbon domain containing 1 & 2.8 & 0.0400 \\
\hline NM_001206196 & KLHL24 & Kelch-like 24 (Drosophila) & 2.6 & 0.0212 \\
\hline NM_001076517 & LY6D & Lymphocyte antigen 6 complex, locus D & 2.5 & 0.0414 \\
\hline NM_001035473 & GK5 & Glycerol kinase 5 & 2.2 & 0.0210 \\
\hline NM_001075890 & KLK10 & Kallikrein-related peptidase 10 & 2.1 & 0.0445 \\
\hline NM_001083791 & SH3BGRL2 & SH3 domain binding glutamic acid-rich protein like 2 & 1.9 & 0.0030 \\
\hline \multicolumn{5}{|l|}{ Down-regulated genes } \\
\hline XM_002685338 & $\mathrm{IFIH} 1$ & Interferon induced with helicase $\mathrm{C}$ domain 1 & 4.0 & 0.0485 \\
\hline NM_001101195 & KCNA2 & Potassium voltage-gated channel, shaker-related subfamily, member 2 & 3.5 & 0.0204 \\
\hline NM_180998 & LTF & Lactotransferrin & 2.9 & 0.0286 \\
\hline NM_001076843 & SLC30A3 & Solute carrier family 30 (zinc transporter), member 3 & 2.6 & 0.0289 \\
\hline NM_001076494 & $\mathrm{C} 8 \mathrm{H} 8 \mathrm{orf13}$ & Chromosome 8 open reading frame 13 ortholog & 2.5 & 0.0406 \\
\hline NM_001105411 & GFRA1 & GDNF family receptor alpha 1 & 2.5 & 0.0383 \\
\hline NM_174018 & CFTR & $\begin{array}{l}\text { Cystic fibrosis transmembrane conductance regulator (ATP-binding cassette sub-family } \\
\text { C, member } 7 \text { ) }\end{array}$ & 2.5 & 0.0316 \\
\hline NM_001077941 & MARCH3 & Membrane-associated ring finger (C3HC4) 3 & 2.5 & 0.0158 \\
\hline NM_173959 & SCD & Stearoyl-CoA desaturase (delta-9-desaturase) & 2.0 & 0.0096 \\
\hline NM_174602 & SLC2A1 & Solute carrier family 2 (facilitated glucose transporter), member 1 & 1.9 & 0.0057 \\
\hline
\end{tabular}

Gene expression profiles of CAR and ICAR in contralateral uterine horns

A total of 443 and 257 genes were differentially expressed in CAR and ICAR of the contralateral uterine horn of RB cows, respectively when compared with nonRB cows (adjusted $P$-value $<0.05$, fold-change $>1.0$ ). All data of individual gene changes in CAR and ICAR are available in Additional file 1: Tables S3 and S4, respectively. Out of these, 333 genes were upregulated and 110 genes were down-regulated in CAR, whereas 121 genes were up-regulated and 136 genes were down-regulated in ICAR. The top 10 upand down-regulated known genes in CAR are shown in Table 6. Similar to CAR of the ipsilateral side, the most pronounced up-regulated gene in $R B$ cows was GSTA3 (Glutathione $S$-transferase, alpha 3; 12.7-fold). The most down-regulated gene in RB cows was SLC39A2 (Solute carrier family 39 (zinc transporter), member 2; 2.7-fold). Table 7 shows the top five functional annotations of DEG in CAR between RB and non-RB cows. Biological functions of positive regulation of biological process, positive regulation of cellular process, organ morphogenesis, anatomical structure morphogenesis, and anatomical structure development were highly enriched in up-regulated genes, whereas biological functions of regulation of protein kinase activity, regulation of kinase activity, regulation of transferase activity and carboxylic acid metabolic process were highly enriched in down-regulated genes.

Table 9 Top 5 functional annotations of up- and down-regulated genes in ICAR of contralateral uterine horns

\begin{tabular}{lll}
\hline Term & Count & $P$-value \\
\hline Up-regulated genes & & \\
GO:0010467 gene expression & 17 & 0.0004 \\
GO:0080090 regulation of primary metabolic process & 19 & 0.0013 \\
GO:0060255 regulation of macromolecule & 19 & 0.0015 \\
metabolic process & & \\
GO:0008152 metabolic process & 38 & 0.0033 \\
GO:0019222 regulation of metabolic process & 19 & 0.0040 \\
Down-regulated genes & & \\
GO:0044238 primary metabolic process & 34 & 0.0023 \\
GO:0006810 transport & 17 & 0.0025 \\
GO:0051234 establishment of localization & 17 & 0.0026 \\
GO:0051179 localization & 18 & 0.0027 \\
GO:0008152 metabolic process & 35 & 0.0028 \\
\hline
\end{tabular}


Table 10 Up- and down-regulated known genes in whole uterus of RB cows as compared with non-RB cows

\begin{tabular}{|c|c|c|c|c|}
\hline GenBank accession ID & Gene symbol & Gene description & Fold change & $P$-value \\
\hline \multicolumn{5}{|l|}{ Up-regulated genes } \\
\hline NM_174690 & PRSS2 & Protease, serine, 2 (trypsin 2) & 12.3 & 0.0018 \\
\hline NM_001077112 & GSTA3 & Glutathione S-transferase, alpha 3 & 6.7 & 0.0002 \\
\hline NM_001014878 & PIPOX & Pipecolic acid oxidase & 6.4 & $<0.0001$ \\
\hline NM_174278 & CNGA1 & Cyclic nucleotide gated channel alpha 1 & 4.3 & 0.0024 \\
\hline S82409 & IGG1C & IgG1 heavy chain constant region & 3.7 & 0.0081 \\
\hline BC112657 & Vl1a & Immunoglobulin lambda light chain variable region & 3.7 & 0.0076 \\
\hline S82407 & IgCgamma & IgG2a heavy chain constant region & 3.4 & 0.0347 \\
\hline NM_001025346 & DAPL1 & death associated protein-like 1 & 3.4 & 0.0075 \\
\hline NM_001080353 & $\mathrm{PI} 3$ & Peptidase inhibitor 3, skin-derived (SKALP) & 3.2 & 0.0022 \\
\hline NM_001166616 & C5 & Complement component 5 & 2.8 & 0.0044 \\
\hline NM_001024569 & ELF5 & E74-like factor 5 (ets domain transcription factor) & 2.8 & 0.0047 \\
\hline NM_001075910 & CCDC113 & Coiled-coil domain containing 113 & 2.7 & 0.0432 \\
\hline NM_173945 & NTS & Neurotensin & 2.6 & $<0.0001$ \\
\hline NM_001034351 & TNNC1 & Troponin C type 1 (slow) & 2.5 & 0.0004 \\
\hline NM_001206196 & KLHL24 & Kelch-like 24 (Drosophila) & 2.5 & $<0.0001$ \\
\hline NM_001046400 & ZNRD1 & zinc ribbon domain containing 1 & 2.3 & $<0.0001$ \\
\hline NM_001193109 & SDCCAG8 & Serologically defined colon cancer antigen 8 & 2.2 & 0.0001 \\
\hline NM_174010 & CD36 & CD36 molecule (thrombospondin receptor) & 2.2 & 0.0073 \\
\hline XM_588022 & SPOPL & Speckle-type POZ protein-like & 2.2 & $<0.0001$ \\
\hline NM_173880 & $\mathrm{H} 4$ & Histone H4 & 2.1 & 0.0033 \\
\hline NM_001098155 & ZNF322A & Zinc finger protein $322 \mathrm{~A}$ & 2.1 & 0.0005 \\
\hline NM_001035380 & GC & Group-specific component (vitamin D binding protein) & 2.0 & 0.0269 \\
\hline NM_001035473 & GK5 & Glycerol kinase 5 & 2.0 & 0.0003 \\
\hline \multicolumn{5}{|l|}{ Down-regulated genes } \\
\hline NM_181005 & CHGA & Chromogranin A (parathyroid secretory protein 1) & 3.9 & 0.0005 \\
\hline NM_001076073 & KRT35 & Keratin 35 & 3.3 & 0.0011 \\
\hline NM_001034728 & THBS4 & Thrombospondin 4 & 3.2 & $<0.0001$ \\
\hline NM_001206057 & CPXM2 & Carboxypeptidase X (M14 family), member 2 & 3.1 & $<0.0001$ \\
\hline NM_001143735 & PRF1 & Perforin 1 (pore forming protein) & 3.0 & 0.0090 \\
\hline NM_001002763 & $\mathrm{CDH} 1$ & Cadherin 1, type 1, E-cadherin (epithelial) & 2.9 & 0.0097 \\
\hline NM_176851 & FUT5 & Fucosyltransferase 5 (alpha $(1,3)$ fucosyltransferase) & 2.7 & 0.0038 \\
\hline XM_002685338 & $\mathrm{IFIH} 1$ & Interferon induced with helicase $\mathrm{C}$ domain 1 & 2.5 & 0.0040 \\
\hline NM_001081734 & MOCS3 & Molybdenum cofactor synthesis 3 & 2.5 & 0.0465 \\
\hline NM_174039 & DPP4 & Dipeptidyl-peptidase 4 & 2.4 & 0.0158 \\
\hline NM_001102080 & CSNK1D & Casein kinase 1, delta & 2.3 & 0.0144 \\
\hline NM_001102060 & TBC1D10C & TBC1 domain family, member $10 \mathrm{C}$ & 2.3 & 0.0391 \\
\hline NM_001081539 & $\mathrm{C} 11 \mathrm{H} 2 \mathrm{orf} 49$ & Chromosome 11 open reading frame, human C2orf49 & 2.3 & 0.0354 \\
\hline AF068848 & VpreB & Surrogate light chain & 2.3 & 0.0204 \\
\hline NM_001127317 & $\mathrm{MIC} 1$ & Major histocompatibility class I related protein & 2.2 & 0.0135 \\
\hline NM_205801 & CLDN3 & Claudin 3 & 2.2 & 0.0196 \\
\hline NM_001077887 & CLASRP & CLK4-associating serine/arginine rich protein & 2.2 & 0.0245 \\
\hline NM_174513 & ADAP1 & ArfGAP with dual PH domains 1 & 2.1 & 0.0169 \\
\hline NM_001105478 & SSLP1 & Secreted seminal-vesicle Ly- 6 protein 1 & 2.1 & 0.0004 \\
\hline
\end{tabular}


Table 10 Up- and down-regulated known genes in whole uterus of RB cows as compared with non-RB cows (Continued)

\begin{tabular}{lllll}
\hline NM_001077962 & STAC & SH3 and cysteine rich domain & 2.1 & $<0.0001$ \\
XM_002687754 & PLEKHA5 & Pleckstrin homology domain containing, family A member 5 & 2.1 & 0.0003 \\
NM_001101239 & GRP & Gastrin-releasing peptide & 2.1 & 0.0059 \\
NM_001205648 & SLC39A2 & Solute carrier family 39 (zinc transporter), member 2 & 2.0 & 0.0001 \\
\hline
\end{tabular}

Table 8 shows the top 10 up- and down-regulated known genes in ICAR. The highest increase and decrease in gene expression in $\mathrm{RB}$ cows were found for PIPOX (Pipecolic acid oxidase; 8.8-fold) and IFIH1 (Interferon induced with helicase C domain 1; 4.0-fold), respectively. The top five functional annotations of DEG in the ICAR of contralateral uterine horns between RB and non-RB cows are listed in Table 9. The GOs containing genes regulating gene expression, regulation of primary metabolic process, regulation of macromolecule metabolic process, metabolic process and regulation of metabolic process were highly enriched in up-regulated genes. In down-regulated genes, the GO terms involved in primary metabolic process, transport, establishment of localization, localization and metabolic process were highly enriched.

\section{Gene expression profiles of whole uterus}

To characterize differential global gene expression profiles in the endometrium of RB and non-RB cows not only locally in each endometrial compartment but also globally in the uterus, we also performed bioinformatics analysis by combining the microarray gene data sets of four endometrial compartments in each cow as whole uterus. A total of 76 genes were found to be differentially expressed in the whole uterus of $\mathrm{RB}$ cows when compared with non-RB cows (adjusted $P$-value $<0.05$, fold-change $>2.0$ ). Among these, 37 genes were upregulated and 39 genes were down-regulated. All upand down-regulated known genes in the whole uterus are shown in Table 10. The most pronounced upand down-regulated gene expression in RB cows was found for PRSS2 (Protease, serine, 2 (trypsin 2); 12.3-fold) and CHGA (Chromogranin A (parathyroid secretory protein 1); 3.9-fold), respectively.

\section{Validation of gene expression by qPCR}

We selected the top two and top five up- and downregulated known genes in each endometrial compartment and whole uterus between RB and non-RB cows, respectively to validate the changes in gene expression obtained from microarray analysis by qPCR. qPCR analysis clearly confirmed the microarray results in each endometrial compartment except for FAM83D (Fig. 1h), SLC39A2 (Fig. 2c), PLEKHA5 (Fig. 2d) and IFIH1 (Fig. 2g). In the whole uterus, the microarray results were confirmed except for PRF1 (Fig. 3j).
Protein localization of CHGA, GSTA3 and PRSS2 in the endometrium of RB and non-RB cows

Figure 4 shows the results of immunohistochemistry for CHGA, GSTA3 and PRSS2 in the endometrial tissues of ipsilateral uterine horns of $\mathrm{RB}$ and non- $\mathrm{RB}$ cows on Day 15 of the estrous cycle. In both RB and non-RB cows, a distinct CHGA signal was found in the uterine luminal epithelium and a part of uterine stroma under the epithelium (Fig. 4a and c). CHGA protein was also detected moderately in the glandular epithelium in both $\mathrm{RB}$ and non- $\mathrm{RB}$ cows and in the uterine stroma in $\mathrm{RB}$ cows (Fig. 4b and d). A positive GSTA3 signal was detected in the uterine luminal, uterine stroma and glandular epithelium in RB cows (Fig. 4e and f), whereas positive staining was not observed in non-RB cows (Fig. 4g and h). PRSS2 protein was moderately detected in the uterine luminal epithelium and glandular epithelium, and partially intense staining was observed in the uterine stroma under the epithelium in both RB and non-RB cows (Fig. 4i,j, ,k and l).

\section{Discussion}

This is the first study to investigate global gene expression profiles of endometrium between $\mathrm{RB}$ and non-RB cows in both each endometrial compartments and the whole uterus. As we hypothesized, the microarray analysis identified a number of characteristic up- and down-regulated genes specific to each of four endometrial compartments of $\mathrm{RB}$ cows. The RB cows used in this study had experienced pregnancy and then became infertile. Thus, long-term infertility in the RB cows may be associated with alteration of endometrial function. Our results support that alteration of uterine environment, which may be induced by changes in the endometrial gene expression, could be a possible involvement of low fertility in the RB cattle.

Even though the endometrial gene expression profiles were regionally different in the endometrial compartments, GSTA3 was identified as the most pronounced up-regulated gene in the CAR of both ipsilateral and contralateral uterine horn. GSTA3 is a member of the class Alpha GST isoenzymes which exert a critical role in the detoxification of electrophilic decomposition products generated by reactive oxygen species (ROS) and metabolism of xenobiotics through glutathione conjugation with electrophilic compounds [34-37]. Similar to our results, a recent study has demonstrated that cows with low endometrial receptivity of the embryo 


\section{Ipsilateral uterine horns}

CAR

A

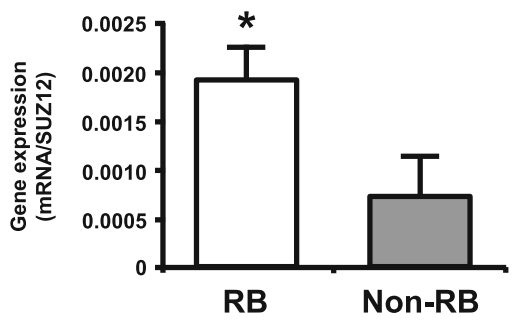

KLHL24

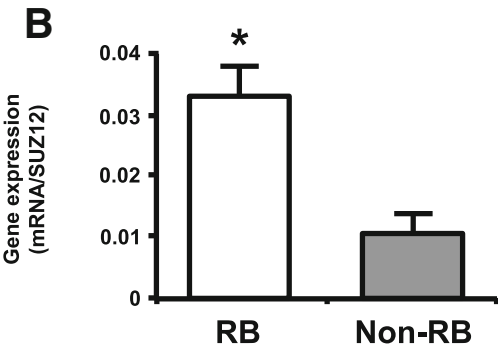

C

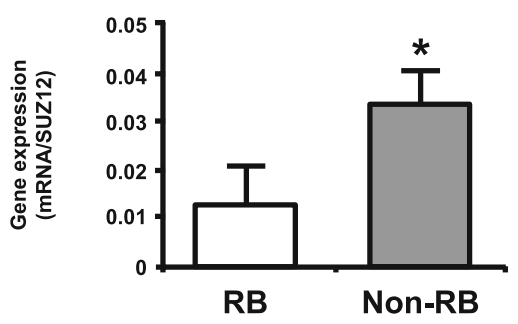

D

KRT35

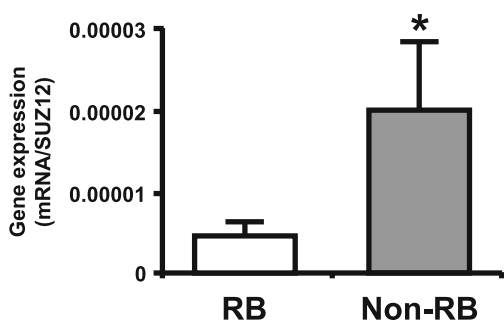

ICAR

E

LPLUNC1

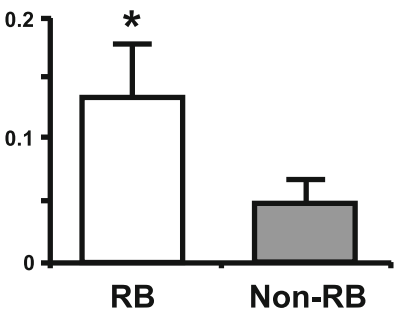

FMO2

$\mathbf{F}$

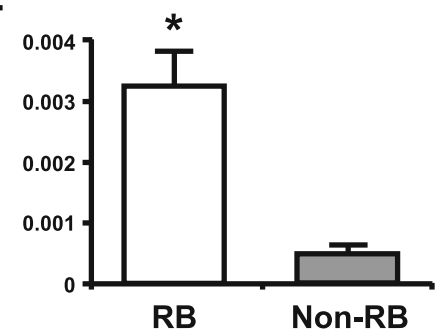

G

THBS4

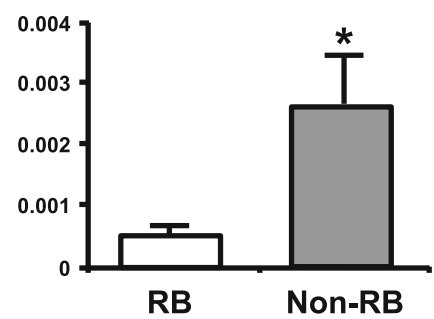

H

FAM83D

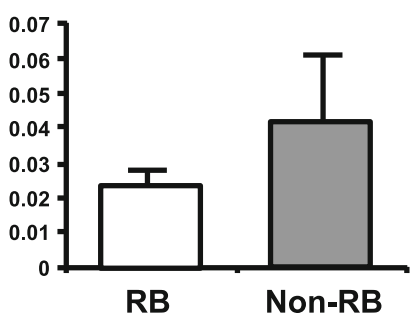

Fig. 1 qPCR analysis of top two up- and down-regulated known genes in ipsilateral uterine horns between RB and non-RB cows for validation of the gene expression changes obtained from microarray analysis. $\mathbf{a}, \mathbf{b}, \mathbf{c}$ and $\mathbf{d} C A R$ and $\mathbf{e}, \mathbf{f}, \mathbf{g}$ and $\mathbf{h} I C A R$. $\mathbf{a}, \mathbf{b}$, e and $\mathbf{f}$ up-regulated known genes in RB cows when compared with non-RB cows. $\mathbf{c}, \mathbf{d}, \mathbf{g}$ and $\mathbf{h}$ ) down-regulated known genes in RB cows when compared with non-RB cows. The expression of mRNA was normalized to the expression of SUZ12 measured in the same RNA preparation. Data are shown as the mean \pm SEM. Asterisks show significant differences $(P<0.05)$ 


\section{Contralateral uterine horns}

CAR

A

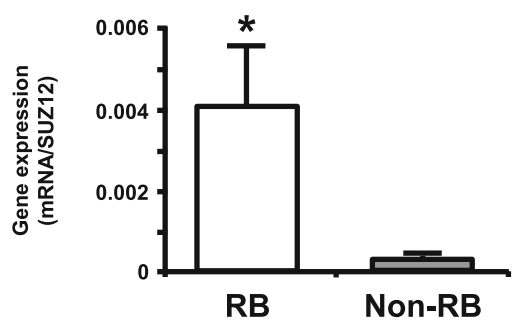

B

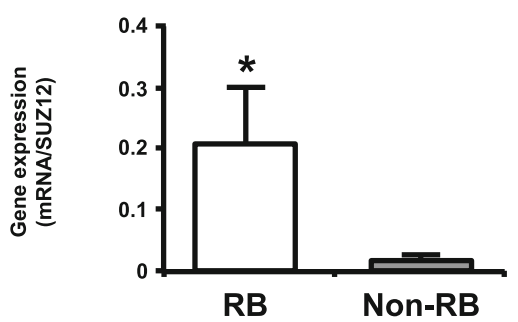

C

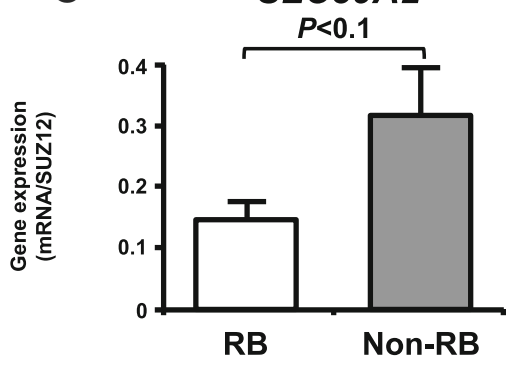

D

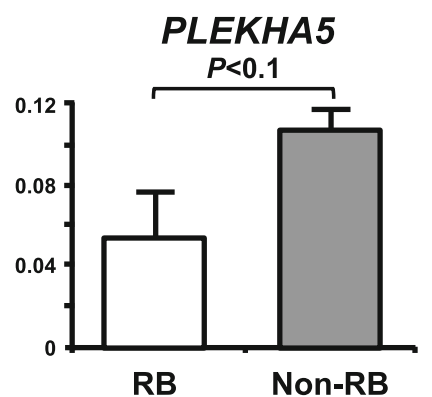

E

ICAR

PIPOX

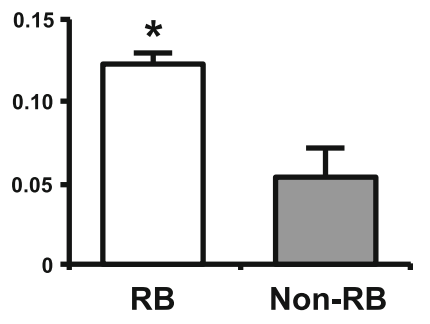

$\mathbf{F}$

CNGA1

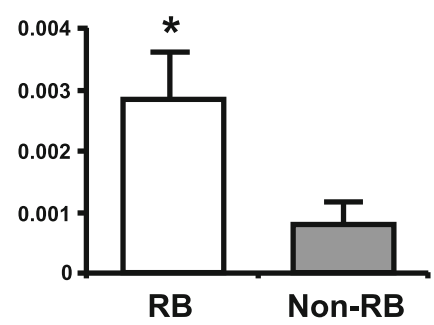

G

IFIH1

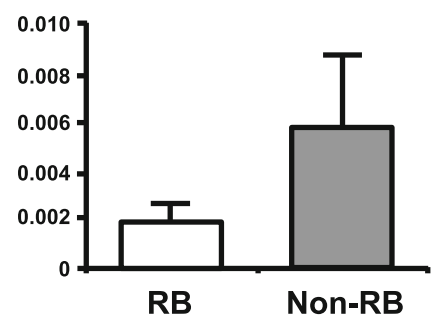

H

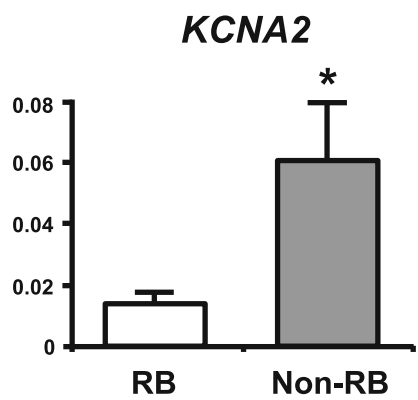

Fig. 2 qPCR analysis of top two up- and down-regulated known genes in contralateral uterine horns between RB and non-RB cows for validation of the gene expression changes obtained from microarray analysis. $\mathbf{a}, \mathbf{b}, \mathbf{c}$ and $\mathbf{d}$ CAR and $\mathbf{e}, \mathbf{f}, \mathbf{g}$ and $\mathbf{h} I C A R$. $\mathbf{a}, \mathbf{b}, \mathbf{e}$ and $\mathbf{f}$ up-regulated known genes in RB cows when compared with non-RB cows. $\mathbf{c}, \mathbf{d}, \mathbf{g}$ and $\mathbf{h}$ down-regulated known genes in RB cows when compared with non-RB cows. The expression of mRNA was normalized to the expression of SUZ12 measured in the same RNA preparation. Data are shown as the mean \pm SEM. Asterisks show significant differences $(P<0.05)$ 

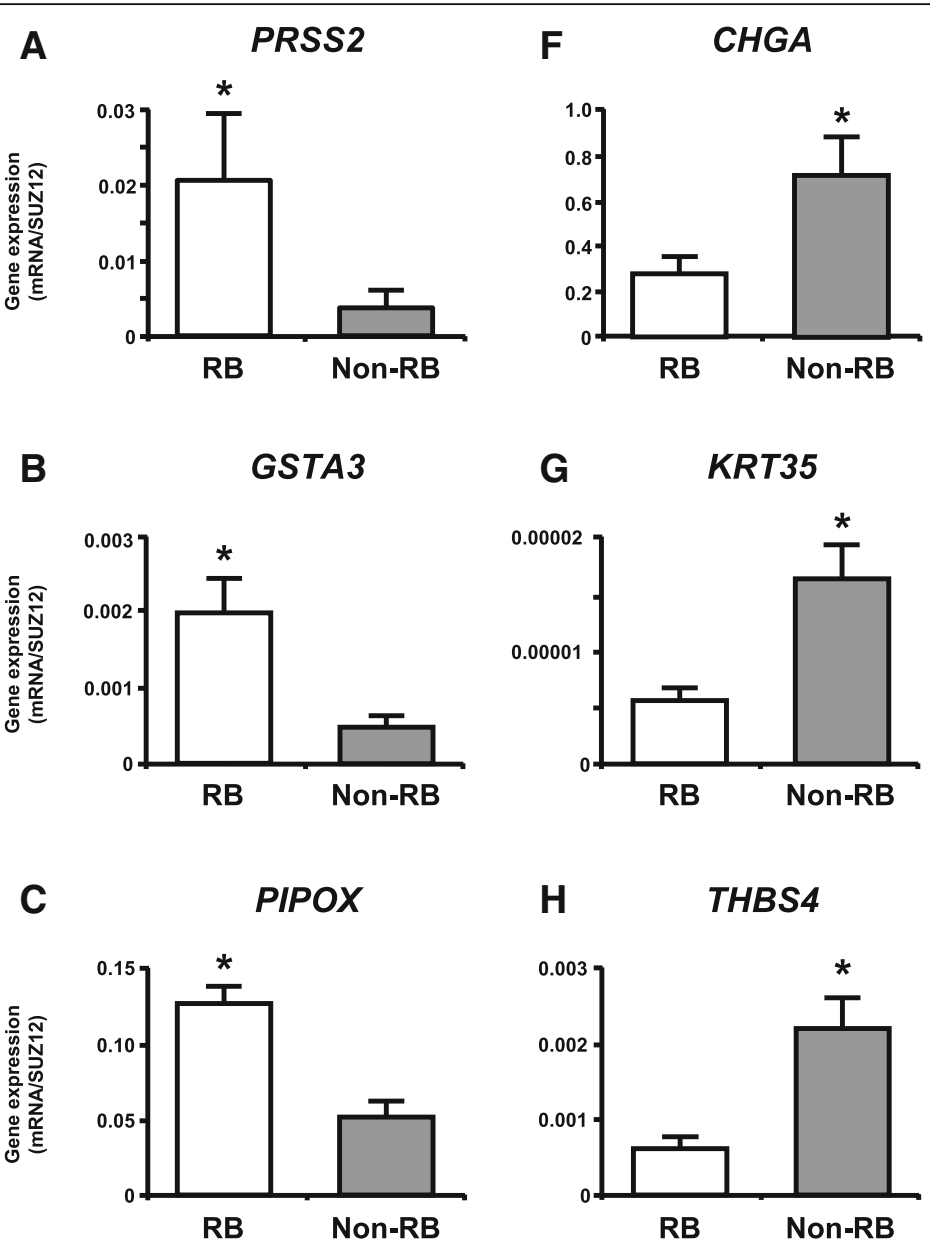

D
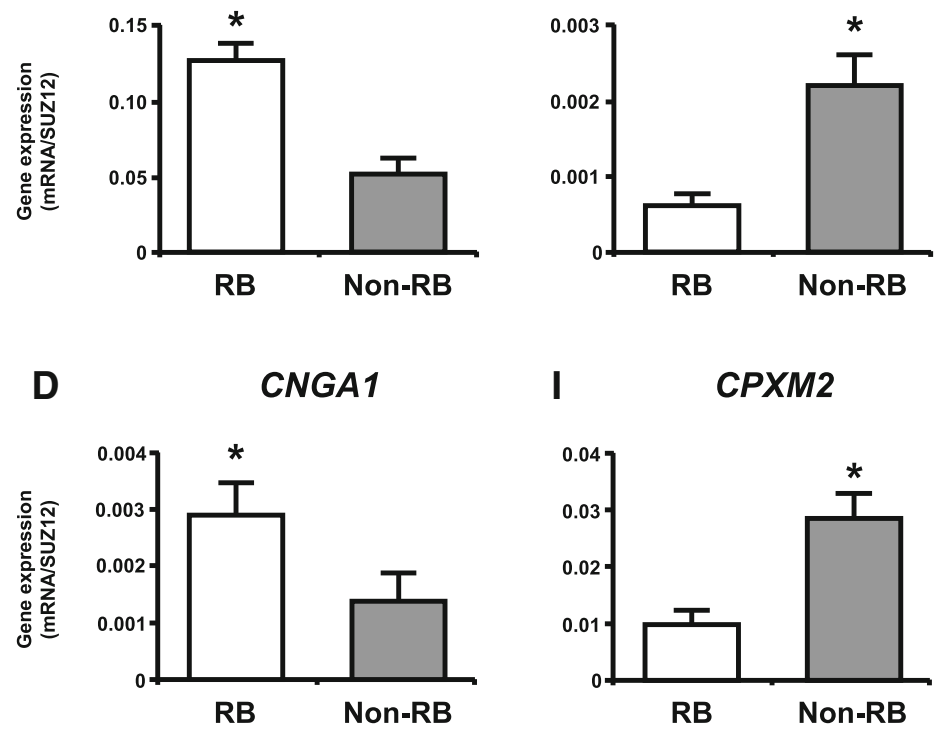

E
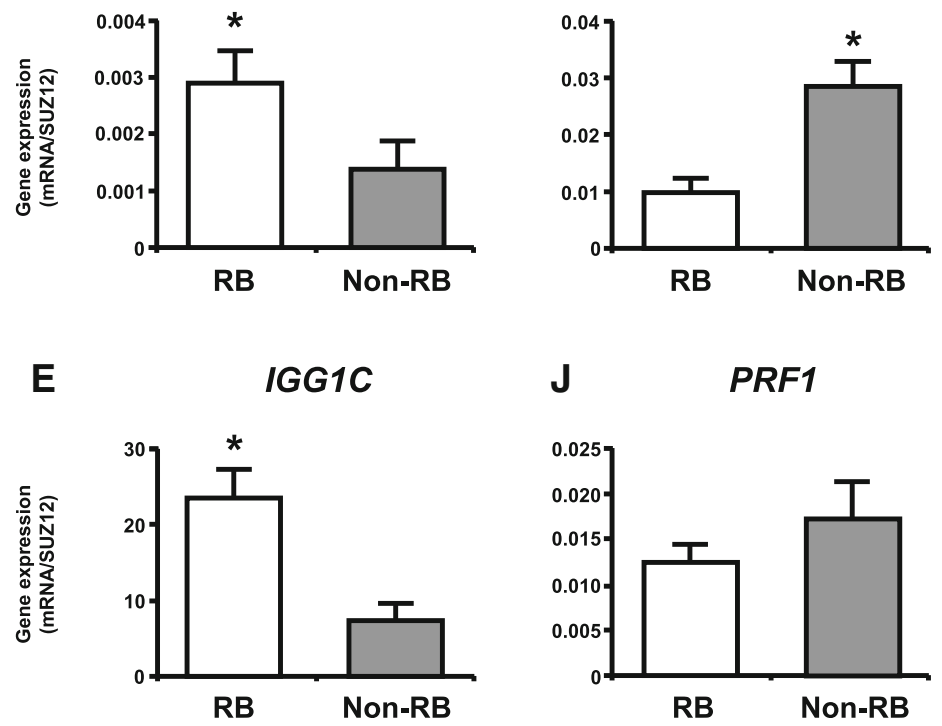

Fig. 3 (See legend on next page.) 
(See figure on previous page.)

Fig. 3 qPCR analysis of top five up- and down-regulated known genes in whole uterus between RB and non-RB cows for validation of the gene expression changes obtained from microarray analysis. $\mathbf{a}, \mathbf{b}, \mathbf{c}, \mathbf{d}$, e up-regulated known genes in RB cows when compared with non-RB cows. $\mathbf{f}, \mathbf{g}, \mathbf{h}, \mathbf{i}, \mathbf{j}$ down-regulated known genes in RB cows when compared with non-RB cows. The expression of mRNA was normalized to the expression of SUZ12 measured in the same RNA preparation. Data are shown as the mean \pm SEM. Asterisks show significant differences $(P<0.05)$

show a higher expression of several oxidative stressresponse genes in the endometrium compared with highly receptive cows at Day 7 of the estrous cycle [7]. Both oxidative stress and xenobiotics are directly responsible for not only an increase in embryonic mortality but also an alteration of uterine function inducing severe gynecological diseases such as endometriosis and preeclampsia [38-42]. We suppose that the CAR of RB cows may be accompanied by enhanced detoxification and elimination of ROS and xenobiotics. Another important contribution of GSTA3 isomerase is in the biosynthesis of steroids, especially testosterone and $\mathrm{P} 4$ in active steroidogenic tissues [43]. Progesterone inhibits endometrial epithelial cell proliferation, adenogenesis and uterine gland development [44, 45]. A previous study showed that RB cows had higher concentrations of $\mathrm{P} 4$ receptor in the endometrium than non- $\mathrm{RB}$ cows, implying the existence of a local hormonal imbalance in RB cows [46]. In the present study, the GSTA3 was also highly expressed in the ICAR of RB cows compared with non-RB cows. In addition, immunohistochemistry revealed that a strong signal of GSTA3 protein was detected in the uterine luminal and glandular epithelium and stroma in RB cows. GSTA3 may also be involved in ICAR functions in RB cows by mediating steroidogenesis.

Gene ontology analysis using DAVID revealed that a number of biological processes and functions were different between RB and non-RB cows in both CAR and ICAR. In the CAR of RB cows, genes involved in development and morphogenesis were mainly up-regulated.

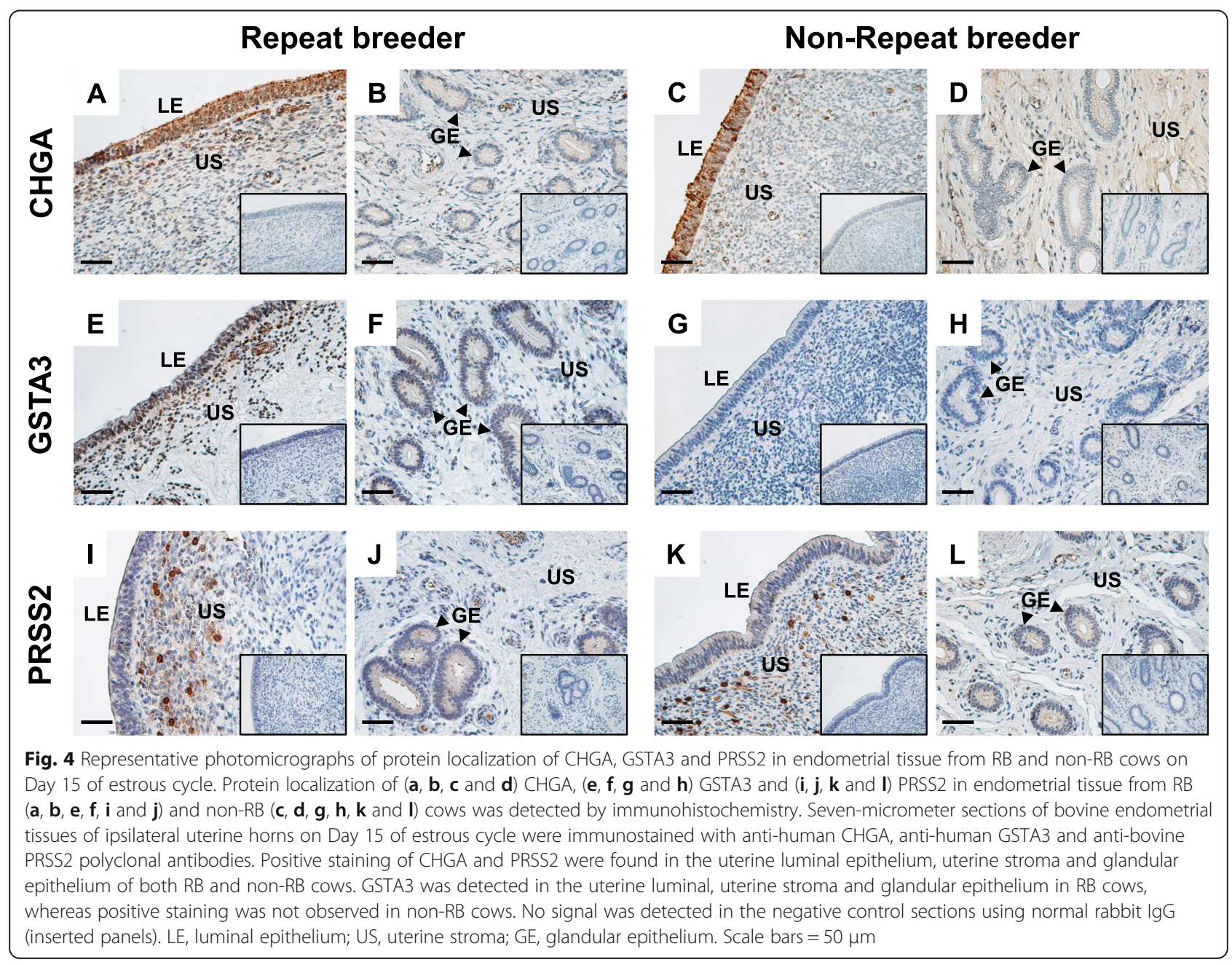


These genes included 14 and 9 genes regulating embryo development and vasculature development, respectively. The CAR eventually attaches with the trophoblast to give rise to the maternal side of the placentome in pregnant animals [22, 23]. Up-regulation of the genes involved in embryo and vasculature development in the CAR may contribute to the success of implantation and following placental formation at the maternal-fetal interface. An increase in the regulation of these genes in the CAR may be one of the characteristics of the RB uterus. In the ICAR of both the ipsilateral and contralateral uterine horns, genes related to metabolic processes were predominantly enriched in both up- and down-regulated genes in $\mathrm{RB}$ cows compared with non-RB cows. The ICAR is a specific compartment containing the uterine glands, which synthesize and secrete various metabolites and histotroph required for estrous cyclicity or development of the conceptus [24]. Alterations of endometrial metabolic processes in RB cows may seriously affect maintenance of uterine function.

The DAVID analysis also revealed that the CAR of the ipsilateral uterine horn of $\mathrm{RB}$ cows is characterized by down-regulation of a number of genes associated with cytoskeleton organization, cell adhesion and cellular component organization compared with non-RB cows. Previous global gene expression studies in bovine endometrium showed that profiles of the genes assigned to these functional categories changes during estrous cycle and peri-implantation [11-13], suggesting that these biological functions may be responsible for the regulation of uterine environment. Additionally, the endometrial cell adhesion molecules play a role in conceptus-endometrium attachment at implantation. A direct comparison of cyclic and pregnant endometrium found cell adhesion and cytoskeleton organization molecules affected by pregnancy in both CAR and ICAR [13]. Around the implantation period, the ipsilateral uterine horn is the site of first occurrence of conceptus-endometrial contact and modification of cytological character was seen exclusively on the CAR $[47,48]$. Therefore, the lower expression of genes regulating cytoskeleton organization and cell adhesion in CAR of $\mathrm{RB}$ cows may be associated with inadequate endometrial responsiveness resulting in implantation failure.

CPXM2 was included in the top 10 down-regulated genes in both CAR and ICAR of the ipsilateral uterine horn. Previous microarray studies found no differences in CPXM2 expression in the bovine endometrium between highly fertile and poor fertile, and between highly fertile and subfertile cows at Day 14 of the estrous cycle [9], while expression decreasing at Day 7 compared to Day 3 of estrus in cows with low embryo receptivity [7]. CPXM2 is assumed to be more sensitive to P4 or some $\mathrm{CL}$ factors in a poorly fertile endometrium that includes the RB. Although the specific roles of CPXM2 remain unknown, DAVID analysis has assigned it belongs to the biological process of proteolysis and cell adhesion. Thus, CPXM2 may be related to alteration of endometrial cell adhesion in RB cows, as well as to the above described cell adhesion related genes that are down-regulated in the CAR of the ipsilateral uterine horn of RB cows.

KLHL24 (Kelch-like 24) was the only gene included in the top 10 up-regulated genes in all four endometrial compartments. A member of the KLHL family including KLHL24 is known to be involved in ubiquitination $[49,50]$. It has been reported that lower expression of genes associated with ubiquitination in high fertile as compared with subfertile cows [9]. Although the specific roles of KLHL24 have not yet been elucidated, an increase in oxidative stress stimulated KLHL24 expression in human fibroblast cells [51], leading us to speculate that this gene is up-regulated to counteract cytoskeleton destruction by ROS- induced cell damage and/or to degrade proteins in cells exposed to ROS by ubiquitination reaction. Therefore, high expression of KLHL24 in RB cows compared with non-RB cows support the possibility that the endometrium of $\mathrm{RB}$ cows is under oxidative stress. However, it has been reported that the level of KLHL24 gene expression at Day 14 of the estrous cycle shows no significant difference among high fertile, low fertile and infertile cows [9]. The functional contribution of endometrial KLHL24 in bovine fertility remains unclear.

Analysis of the combined gene data sets of the four endometrial compartments revealed gene expression profiles of the whole uterus. PRSS 2 and CHGA were the most pronounced up- and down-regulated genes, respectively. PRSS2 is a member of the trypsin family of serine proteases and degrades type I collagen directly or indirectly by activating several procollagenolytic matrix metalloproteinases (MMPs) [52, 53]. CHGA works as a pro-hormone for pancreastatin, vasostatin and catestatin [54-56]. Full-length CHGA and vasostatin act as antiangiogenic factors to inhibit two potent angiogenic factors, basic fibroblast growth factor (bFGF) and vascular endothelial growth factor, while CHGA cleaved by thrombin and catestatin promote angiogenesis by inducing the release of bFGF from vascular endothelial cells [57]. In the present study, we found that both PRSS2 and CHGA proteins were localized in the luminal and glandular epithelium and in the stroma of the endometrium. These localizations coincide with the tissue site of gelatinase activity of MMP-2 and the localization of MMPs and bFGF in the bovine endometrium [58-60], suggesting paracrine and autocrine actions of PRSS2 and CHGA with MMPs and bFGF in the bovine endometrium. In addition, genes involved in cell death (DAPL1 and PRF1) or cell attachment (CD36, CDH1, CPXM2, KRT35 and THBS4) were also differentially expressed between RB and non-RB cows. Although 
further studies are needed to clarify, the endometrium of RB cows might not only be involved in the promotion of tissue remodeling and imbalance of angiogenesis but also in the degradation of cell renewal and tissue structure.

In cattle, around Day 15 of pregnancy is a stage of the beginning of conceptus elongation and maternal recognition of pregnancy [26]. A recent RNA-seq study identified numerous conceptus-expressed ligands that interact with corresponding receptors expressed on the endometrium and vice versa at Day 16 of pregnancy in cattle [61]. In the present study, some genes of endometrium expressed ligands (CCL4, CCL14, COL1A2, EDN1, F2, MMP2, THBS4 and TIMP3) and receptors $(A C V R 2 B$, BMPR2, CD4, CD36, IGF2R, IL1ORB, KDR, TNFRSF 25 and $V L D L R$ ) that interact with conceptus reported by Mamo et al. were differentially expressed between RB and non-RB cows. In addition, other genes encoding growth factors (FGF9 and GDF7) and cytokines (CCL8, $C D 14$ and $C D 53)$ were down-regulated in the RB cows as compared with non-RB cows. Although the functional role of these two growth factors in bovine endometrium remains to be elucidated, FGF9 induces endometrial stromal cell proliferation [62]. Up-regulation of FGF9 and GDF7 expressions were detected in equine and/or swine pregnant endometrium and may be implicated in embryo-maternal communication at early pregnancy $[63,64]$. The receptors of these growth factors were expressed in not only endometrium but also conceptus at Day 16 of pregnancy in cattle [61]. Therefore, alteration of the expression of these ligands and receptors in the RB cows may affect conceptus development and maternal recognition of pregnancy if a conceptus presents in the RB cows.

\section{Conclusion}

The results of the present study support the hypothesis that endometrial gene expression profiles are different between RB and non-RB cows. In RB cows, characteristic gene expression was identified in both the CAR and ICAR of both ipsilateral and contralateral uterine horns. The enriched GO terms of these genes were related to cell adhesion and morphogenesis in the CAR and metabolism in the ICAR. These results suggest that local regulation of molecular mechanisms in each endometrial compartment may contribute to normal uterine physiology. Therefore, the identified candidate endometrial genes and functions are likely to be involved in bovine reproductive performance. The present study could provide an information base for understanding underlying molecular pathogenesis and developing a treatment of repeat breeding in cattle from the point of view of endometrial function.

\section{Additional file}

\begin{abstract}
Additional file 1: Table S1. List of up- and down-regulated genes in CAR of ipsilateral uterine horns of RB cows $(n=4)$ on Day 15 of the estrous cycle as compared with non-RB cows $(n=4)$. Table S2. List of up- and down-regulated genes in ICAR of ipsilateral uterine horns of RB cows on Day 15 of the estrous cycle as compared with non-RB cows. Table S3. List of up- and down-regulated genes in CAR of contralateral uterine horns of RB cows on Day 15 of the estrous cycle as compared with non-RB cows. Table S4. List of up- and down-regulated genes in ICAR of contralateral uterine horns of RB cows on Day 15 of the estrous cycle as compared with non-RB cows. (XLSX $254 \mathrm{~kb}$ )
\end{abstract}

\section{Abbreviations}

ACVR2B: Activin A receptor type 2B; Al: Artificial insemination; bFGF: Basic fibroblast growth factor; BMPR2: Bone morphogenetic protein receptor type 2; CAR: Caruncular; CCL: Chemokine (C-C motif) ligand; CD4: CD4 molecule; CD14: CD14 molecule; CD36: CD36 molecule; CD53: CD53 molecule; CDH1: Cadherin 1, type 1; CHGA: Chromogranin A; CL: Corpus luteum; COL1A2: Collagen type I alpha 2 chain; CPXM2: Carboxypeptidase X (M14 family), member 2; DAPL1: Death associated protein-like 1; DAVID: Database for annotation, visualization and integrated discovery; DEG: Differentially expressed genes; EDN1: Endothelin 1; F2: Coagulation factor II, thrombin; FAM83D: Protein FAM83D; FGF9: Fibroblast growth factor 9; GDF7: Growth differentiation factor 7; GE: Glandular epithelium; GEO: Gene expression omnibus; GO: Gene ontology; GSTA3: Glutathione S-transferase A3; ICAR: Intercaruncular; IFIH1: Interferon induced with helicase C domain 1; IGF2R: Insulin like growth factor 2 receptor; IL10RB: Interleukin 10 receptor subunit beta; KDR: Kinase insert domain receptor; KLHL24: Kelch-like 24; KRT35: Keratin 35; LE: Luminal epithelium; LPLUNC1: Von Ebner minor salivary gland protein; MMP: Matrix metalloproteinase; P4: Progesterone;

PIPOX: Pipecolic acid oxidase; PLEKHA5: Pleckstrin homology domain containing, family A member 5; PRF1: Perforin 1; PRSS2: Trypsin 2; qPCR: Quantitative real-time RT-PCR; RB: Repeat breeder; ROS: Reactive oxygen species; SLC39A2: Solute carrier family 39 (zinc transporter), member 2; SUZ12: Suppressor of zeste 12; THBS4: Thrombospondin 4; TIMP: Tissue inhibitor of metalloproteinase; TNFRSF25: Tumor necrosis factor receptor superfamily member 25; US: Uterine stroma; VLDLR: Very low density lipoprotein receptor

\section{Acknowledgement}

The authors thank the staff of Livestock Research Support Center of the National Agriculture and Food Research Organization for animal management and their technical assistance for sample collection. This manuscript was proofread by a professional service (SciRevision, Kagawa, Japan) prior to submission.

\section{Funding}

This study was supported by a Grant-in-Aid for Research Program on Innovative Technologies for Animal Breeding, Reproduction, and Vaccine Development (REP1001) from the Ministry of Agriculture, Forestry and Fisheries of Japan.

\section{Availability of data and materials}

All microarray data are available at the Gene Expression Omnibus (GEO) database at NCBI (http://www.ncbi.nlm.nih.gov/geo), under accession numbers GSE79367. All datasets on which the conclusions of the paper rely are available to readers.

\section{Authors' contributions}

KGH participated in the design of the study, collected the materials, carried out all experiments and drafted the manuscript. $\mathrm{MH}$ collected the materials and helped to carry out QPCR and immunohistochemistry. KK carried out microarray and microarray data analysis. $\mathrm{KH}$ and SF carried out immunohistochemistry. TT participated in the design of the study, collected the materials and carried out microarray experiments. RS supervised the study, collected the materials and helped to carry out all experiments. All authors read and approved the final manuscript.

\section{Competing interests}

The authors declare that they have no competing interests. 


\section{Consent for publication}

Not applicable.

\section{Ethics approval and consent to participate}

All procedures in animal experiments were carried out in accordance with guidelines approved by the Animal Ethics Committee of the National Institute of Agrobiological Sciences for the use of animals (permission number: H18-036).

\section{Publisher's Note}

Springer Nature remains neutral with regard to jurisdictional claims in published maps and institutional affiliations.

\section{Author details}

'Division of Animal Breeding and Reproduction Research, Institute of Livestock and Grassland Science, National Agriculture and Food Research Organization, Tsukuba 305-0901, Japan. ${ }^{2}$ Division of Animal Sciences, Institute of Agrobiological Sciences, National Agriculture and Food Research Organization, Tsukuba 305-8602, Japan. ${ }^{3}$ Cooperative Department of Veterinary Medicine, Faculty of Agriculture, Iwate University, Morioka 020-8550, Japan

\section{Received: 14 July 2016 Accepted: 3 March 2017}

Published online: 23 March 2017

\section{References}

1. Perez-Marin CC, Calero GV, Moreno LM. Clinical Approach to the Repeat Breeder Cow Syndrome. In: Perez-Marin CC, editor. A Bird's-Eye View of Veterinary Medicine. INTECH Open Access Publisher; 2012. doi:10.5772/31374.

2. Tanabe TY, Hawk HW, Hasler JF. Comparative fertility of normal and repeatbreeding cows as embryo recipients. Theriogenology. 1985;23:687-96.

3. Dochi O, Takahashi K, Hirai T, Hayakawa H, Tanisawa M, Yamamoto Y, Koyama $\mathrm{H}$. The use of embryo transfer to produce pregnancies in repeat-breeding dairy cattle. Theriogenology. 2008;69:124-8.

4. Gustafsson H, Larsson K. Embryonic mortality in heifers after artificial insemination and embryo transfer: differences between virgin and repeat breeder heifers. Res Vet Sci. 1985:39:271-4

5. Albihn A, Gustafsson H, Rodriguez-Martinez H, Larsson K. Development of day 7 bovine demi-embryos transferred into virgin and repeat-breeder heifers. Anim Reprod Sci. 1989;21:161-76.

6. Katagiri S, Moriyoshi M. Alteration of the endometrial EGF profile as a potential mechanism connecting the alterations in the ovarian steroid hormone profile to embryonic loss in repeat breeders and high-producing cows. J Reprod Dev. 2013:59:415-20.

7. Ponsuksili S, Murani E, Schwerin M, Schellander K, Tesfaye D, Wimmers K. Gene expression and DNA-methylation of bovine pretransfer endometrium depending on its receptivity after in vitro-produced embryo transfer. PLoS One. 2012;7:e42402

8. Walker CG, Littlejohn MD, Mitchell MD, Roche JR, Meier S. Endometrial gene expression during early pregnancy differs between fertile and subfertile dairy cow strains. Physiol Genomics. 2012;44:47-58.

9. Minten MA, Bilby TR, Bruno RG, Allen CC, Madsen CA, Wang Z, Sawyer JE, Tibary A, Neibergs HL, Geary TW, et al. Effects of fertility on gene expression and function of the bovine endometrium. PLoS One. 2013;8:e69444.

10. Killeen AP, Morris DG, Kenny DA, Mullen MP, Diskin MG, Waters SM. Global gene expression in endometrium of high and low fertility heifers during the mid-luteal phase of the estrous cycle. BMC Genomics. 2014;15:234.

11. Bauersachs S, Ulbrich SE, Gross K, Schmidt SE, Meyer HH, Einspanier R, Wenigerkind $\mathrm{H}$, Vermehren M, Blum H, Sinowatz F, Wolf E. Gene expression profiling of bovine endometrium during the oestrous cycle: detection of molecular pathways involved in functional changes. J Mol Endocrinol. 2005;34:889-908.

12. Mitko K, Ulbrich SE, Wenigerkind H, Sinowatz F, Blum H, Wolf E, Bauersachs S. Dynamic changes in messenger RNA profiles of bovine endometrium during the oestrous cycle. Reproduction. 2008;135:225-40.

13. Mansouri-Attia N, Aubert J, Reinaud P, Giraud-Delville C, Taghouti G, Galio L, Everts RE, Degrelle S, Richard C, Hue I, et al. Gene expression profiles of bovine caruncular and intercaruncular endometrium at implantation. Physiol Genomics. 2009:39:14-27.

14. Forde N, Beltman ME, Duffy GB, Duffy P, Mehta JP, O'Gaora P, Roche JF, Lonergan $\mathrm{P}$, Crowe MA. Changes in the endometrial transcriptome during the bovine estrous cycle: effect of low circulating progesterone and consequences for conceptus elongation. Biol Reprod. 2011;84:266-78

15. Forde N, Carter F, Spencer TE, Bazer FW, Sandra O, Mansouri-Attia N, Okumu LA, McGettigan PA, Mehta JP, McBride R, et al. Conceptus-induced changes in the endometrial transcriptome: how soon does the cow know she is pregnant? Biol Reprod. 2011;85:144-56.

16. Bauersachs S, Ulbrich SE, Reichenbach HD, Reichenbach M, Buttner M, Meyer HH, Spencer TE, Minten M, Sax G, Winter G, Wolf E. Comparison of the effects of early pregnancy with human interferon, alpha 2 (IFNA2), on gene expression in bovine endometrium. Biol Reprod. 2012;86:46.

17. Forde N, Duffy GB, McGettigan PA, Browne JA, Mehta JP, Kelly AK, MansouriAttia N, Sandra O, Loftus BJ, Crowe MA, et al. Evidence for an early endometrial response to pregnancy in cattle: both dependent upon and independent of interferon tau. Physiol Genomics. 2012;44:799-810.

18. Spencer TE, Forde N, Dorniak P, Hansen TR, Romero JJ, Lonergan P. Conceptus-derived prostaglandins regulate gene expression in the endometrium prior to pregnancy recognition in ruminants. Reproduction. 2013;146:377-87.

19. Shimizu T, Krebs S, Bauersachs S, Blum H, Wolf E, Miyamoto A. Actions and interactions of progesterone and estrogen on transcriptome profiles of the bovine endometrium. Physiol Genomics. 2010;42A:290-300.

20. Sakumoto R, Hayashi KG, Saito S, Kanahara H, Kizaki K, Iga K. Comparison of the global gene expression profiles in the bovine endometrium between summer and autumn. J Reprod Dev. 2015;61:297-303.

21. King GJ, Atkinson BA, Robertson HA. Development of the bovine placentome during the second month of gestation. J Reprod Fertil. 1979:55:173-80.

22. King GJ, Atkinson BA, Robertson HA. Development of the bovine placentome from days 20 to 29 of gestation. J Reprod Fertil. 1980;59:95-100.

23. King GJ, Atkinson BA, Robertson HA. Development of the intercaruncular areas during early gestation and establishment of the bovine placenta. J Reprod Fertil. 1981;61:469-74.

24. Filant J, Spencer TE. Uterine glands: biological roles in conceptus implantation, uterine receptivity and decidualization. Int J Dev Biol. 2014;58: 107-16.

25. Pope WF, Maurer RR, Stormshak F. Distribution of progesterone in the uterus, broad ligament, and uterine arteries of beef cows. Anat Rec. 1982; 203:245-50.

26. Ealy $A D$, Yang $Q E$. Control of interferon-tau expression during early pregnancy in ruminants. Am J Reprod Immunol. 2009;61:95-106.

27. Dunne LD, Diskin MG, Sreenan JM. Embryo and foetal loss in beef heifers between day 14 of gestation and full term. Anim Reprod Sci. 2000;58:39-44.

28. Berg DK, van Leeuwen J, Beaumont S, Berg M, Pfeffer PL. Embryo loss in cattle between Days 7 and 16 of pregnancy. Theriogenology. 2010;73:250-60.

29. Kizaki K, Shichijo-Kizaki A, Furusawa T, Takahashi T, Hosoe M, Hashizume K. Differential neutrophil gene expression in early bovine pregnancy. Reprod Biol Endocrinol. 2013;11:6.

30. da Huang W, Sherman BT, Lempicki RA. Systematic and integrative analysis of large gene lists using DAVID bioinformatics resources. Nat Protoc. 2009;4:44-57.

31. Ushizawa K, Takahashi T, Hosoe M, Ishiwata H, Kaneyama K, Kizaki K, Hashizume K. Global gene expression analysis and regulation of the principal genes expressed in bovine placenta in relation to the transcription factor AP-2 family. Reprod Biol Endocrinol. 2007;5:17.

32. Walker CG, Meier S, Mitchell MD, Roche JR, Littlejohn M. Evaluation of realtime PCR endogenous control genes for analysis of gene expression in bovine endometrium. BMC Mol Biol. 2009;10:100.

33. Ushizawa K, Takahashi T, Hosoe M, Kizaki K, Hashizume K. Characterization and expression analysis of SOLD1, a novel member of the retrotransposonderived Ly-6 superfamily, in bovine placental villi. PLoS One. 2009;4:e5814.

34. Hayes JD, McLellan LI. Glutathione and glutathione-dependent enzymes represent a co-ordinately regulated defence against oxidative stress. Free Radic Res. 1999;31:273-300.

35. Hayes JD, Flanagan JU, Jowsey IR. Glutathione transferases. Annu Rev Pharmacol Toxicol. 2005;45:51-88.

36. Ilic Z, Crawford D, Vakharia D, Egner PA, Sell S. Glutathione-S-transferase A3 knockout mice are sensitive to acute cytotoxic and genotoxic effects of aflatoxin B1. Toxicol Appl Pharmacol. 2010;242:241-6.

37. Kensler KH, Slocum SL, Chartoumpekis DV, Dolan PM, Johnson NM, llic Z, Crawford DR, Sell S, Groopman JD, Kensler TW, Egner PA. Genetic or pharmacologic activation of Nrf2 signaling fails to protect against aflatoxin 
genotoxicity in hypersensitive GSTA3 knockout mice. Toxicol Sci. 2014;139:293-300.

38. Baranova H, Canis M, Ivaschenko T, Albuisson E, Bothorishvilli R, Baranov V, Malet P, Bruhat MA. Possible involvement of arylamine N-acetyltransferase 2, glutathione S-transferases M1 and $\mathrm{T} 1$ genes in the development of endometriosis. Mol Hum Reprod. 1999:5:636-41.

39. Jackson LW, Schisterman EF, Dey-Rao R, Browne R, Armstrong D. Oxidative stress and endometriosis. Hum Reprod. 2005;20:2014-20.

40. Yokoi R, Hayashi M, Tamura T, Kobayashi K, Kuroda J, Kusama H, Kagami H, Ono T. Embryonic mortality and intrauterine growth retardation (IUGR) associated with placental alterations in pregnant rats treated with methyl methanesulfonate (MMS) at the peri-implantation stage. J Toxicol Sci. 2008:33:585-98.

41. Celi P, Merlo M, Da Dalt L, Stefani A, Barbato O, Gabai G. Relationship between late embryonic mortality and the increase in plasma advanced oxidised protein products (AOPP) in dairy cows. Reprod Fertil Dev. 2011;23:527-33.

42. Loset M, Mundal SB, Johnson MP, Fenstad MH, Freed KA, Lian IA, Eide IP, Bjorge L, Blangero J, Moses EK, Austgulen R. A transcriptional profile of the decidua in preeclampsia. Am J Obstet Gynecol. 2011;204:84. e81-27.

43. Johansson AS, Mannervik B. Human glutathione transferase A3-3, a highly efficient catalyst of double-bond isomerization in the biosynthetic pathway of steroid hormones. J Biol Chem. 2001;276:33061-5.

44. Gray CA, Bazer FW, Spencer TE. Effects of neonatal progestin exposure on female reproductive tract structure and function in the adult ewe. Biol Reprod. 2001;64:797-804.

45. Filant J, Zhou H, Spencer TE. Progesterone inhibits uterine gland development in the neonatal mouse uterus. Biol Reprod. 2012;86:146. 141-149.

46. Almeida Fo AP, Ayalon N, Bartoov B. Progesterone receptors in the endometrium of normal and repeat-breeder cows. Anim Reprod Sci. 1987;14:11-9.

47. Boshier DP. A histological and histochemical examination of implantation and early placentome formation in sheep. J Reprod Fertil. 1969;19:51-61.

48. Spencer TE, Johnson GA, Bazer FW, Burghardt RC. Fetal-maternal interactions during the establishment of pregnancy in ruminants. Soc Reprod Fertil Suppl. 2007;64:379-96.

49. Dhanoa BS, Cogliati T, Satish AG, Bruford EA, Friedman JS. Update on the Kelch-like (KLHL) gene family. Hum Genomics. 2013;7:13.

50. Lin Z, Li S, Feng C, Yang S, Wang H, Ma D, Zhang J, Gou M, Bu D, Zhang T, et al. Stabilizing mutations of KLHL24 ubiquitin ligase cause loss of keratin 14 and human skin fragility. Nat Genet. 2016;48:1508-16.

51. Schweikl H, Hiller KA, Eckhardt A, Bolay C, Spagnuolo G, Stempfl T, Schmalz G Differential gene expression involved in oxidative stress response caused by triethylene glycol dimethacrylate. Biomaterials. 2008;29:1377-87.

52. Sorsa T, Salo T, Koivunen E, Tyynela J, Konttinen YT, Bergmann U, Tuuttila A, Niemi E, Teronen O, Heikkila P, et al. Activation of type IV procollagenases by human tumor-associated trypsin-2. J Biol Chem. 1997;272:21067-74.

53. Moilanen M, Sorsa T, Stenman M, Nyberg P, Lindy O, Vesterinen J, Paju A, Konttinen YT, Stenman UH, Salo T. Tumor-associated trypsinogen-2 (trypsinogen-2) activates procollagenases (MMP-1, $-8,-13$ ) and stromelysin1 (MMP-3) and degrades type I collagen. Biochemistry. 2003;42:5414-20.

54. Tatemoto K, Efendic S, Mutt V, Makk G, Feistner GJ, Barchas JD. Pancreastatin, a novel pancreatic peptide that inhibits insulin secretion. Nature. 1986;324:476-8.

55. Aardal S, Helle KB, Elsayed S, Reed RK, Serck-Hanssen G. Vasostatins, comprising the $\mathrm{N}$-terminal domain of chromogranin $\mathrm{A}$, suppress tension in isolated human blood vessel segments. J Neuroendocrinol. 1993;5:405-12.

56. Mahata SK, O'Connor DT, Mahata M, Yoo SH, Taupenot L, Wu H, Gill BM, Parmer RJ. Novel autocrine feedback control of catecholamine release. A discrete chromogranin a fragment is a noncompetitive nicotinic cholinergic antagonist. J Clin Invest. 1997;100:1623-33.

57. Helle KB, Corti A. Chromogranin A: a paradoxical player in angiogenesis and vascular biology. Cell Mol Life Sci. 2015;72:339-48.

58. Michael DD, Alvarez IM, Ocon OM, Powell AM, Talbot NC, Johnson SE, Ealy AD. Fibroblast growth factor- 2 is expressed by the bovine uterus and stimulates interferon-tau production in bovine trophectoderm. Endocrinology. 2006;147:3571-9.

59. Kizaki K, Ushizawa K, Takahashi T, Yamada O, Todoroki J, Sato T, Ito A, Hashizume K. Gelatinase (MMP-2 and -9) expression profiles during gestation in the bovine endometrium. Reprod Biol Endocrinol. 2008;6:66.
60. Ulbrich SE, Meyer SU, Zitta K, Hiendleder S, Sinowatz F, Bauersachs S, Buttner M, Frohlich T, Arnold GJ, Reichenbach HD, et al. Bovine endometrial metallopeptidases MMP14 and MMP2 and the metallopeptidase inhibitor TIMP2 participate in maternal preparation of pregnancy. Mol Cell Endocrinol. 2011;332:48-57.

61. Mamo S, Mehta JP, Forde N, McGettigan P, Lonergan P. Conceptusendometrium crosstalk during maternal recognition of pregnancy in cattle. Biol Reprod. 2012;87:6. 1-9.

62. Tsai SJ, Wu MH, Chen HM, Chuang PC, Wing LY. Fibroblast growth factor-9 is an endometrial stromal growth factor. Endocrinology. 2002;143:2715-21.

63. Ostrup E, Bauersachs S, Blum H, Wolf E, Hyttel P. Differential endometrial gene expression in pregnant and nonpregnant sows. Biol Reprod. 2010;83:277-85.

64. Klein C. Novel equine conceptus-endometrial interactions on day 16 of pregnancy based on RNA sequencing. Reprod Fertil Dev. 2016;28:1712-20.

\section{Submit your next manuscript to BioMed Central and we will help you at every step:}

- We accept pre-submission inquiries

- Our selector tool helps you to find the most relevant journal

- We provide round the clock customer support

- Convenient online submission

- Thorough peer review

- Inclusion in PubMed and all major indexing services

- Maximum visibility for your research

Submit your manuscript at www.biomedcentral.com/submit
Biomed Central 